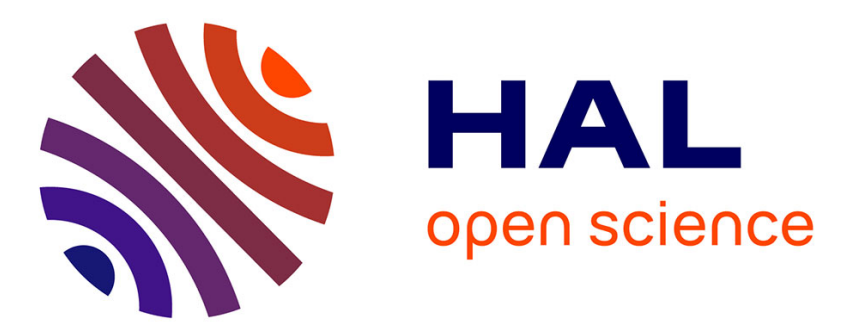

\title{
Observers with dual spatially separated sensors for enhanced estimation: Industrial, automotive, and biomedical applications
}

Rajesh Rajamani, Yan Wang, Garrett D. Nelson, Ryan Madson, Ali Zemouche

\section{- To cite this version:}

Rajesh Rajamani, Yan Wang, Garrett D. Nelson, Ryan Madson, Ali Zemouche. Observers with dual spatially separated sensors for enhanced estimation: Industrial, automotive, and biomedical applications. IEEE Control Systems, 2017, 37 (3), pp.42-58. 10.1109/MCS.2017.2674439 . hal01534700

\author{
HAL Id: hal-01534700 \\ https://hal.science/hal-01534700
}

Submitted on 15 Jan 2018

HAL is a multi-disciplinary open access archive for the deposit and dissemination of scientific research documents, whether they are published or not. The documents may come from teaching and research institutions in France or abroad, or from public or private research centers.
L'archive ouverte pluridisciplinaire HAL, est destinée au dépôt et à la diffusion de documents scientifiques de niveau recherche, publiés ou non, émanant des établissements d'enseignement et de recherche français ou étrangers, des laboratoires publics ou privés. 


\title{
Observers with Dual Spatially Separated Sensors
}

\author{
for Enhanced Estimation
}

\section{Industrial, Automotive and Biomedical Applications}

\author{
Rajesh Rajamani, Yan Wang, Garrett Nelson, Ryan Madson and Ali Zemouche \\ POC: R. Rajamani, rajamani@umn.edu
}

July 14, 2016

The presence of two eyes, ears and nostrils endows mammals with many benefits. These go beyond just having a spare sensory organ. The spatial separation between two similar sensory organs enables enhanced sensory perception. For instance, in the case of the eyes, it is well known that the presence of two spatially separated eyes enables stereopsis or three-dimensional depth perception [1]. This is important for tasks requiring spatial discrimination such as threading a needle, judging a space between one's car and other cars on a road, and also for many sports. Other less well-known benefits of laterally separated eyes include the following:

- $\quad$ Neurologically based binocular summation in the brain increases the signal-to-noise ratio (SNR), increasing detectability of objects, especially under poor illumination and contrast. Night driving would be extremely difficult with one eye [2], [3], [4].

- Spatial localization of objects is done visually with respect to the body. With a single eye, spatial localization would be of an oculocentric nature and would constantly change with eye movements instead of being body-based and stable [3].

- Dual eyes help us see through clutter. This is important for humans who live in cluttered environments and for mammals in general that live in leafy cluttered jungles [5].

Similarly, having two nostrils improves signal-to-noise ratio and also gives the body the ability to simultaneously clean up the air filter in one nostril while the other nostril is used for breathing [6]. 
Having two ears provides both bilateral and binaural benefits [8]. The bilateral benefit is the ability to listen with the ear that has a better SNR. The binaural ability is the ability to combine sounds from the two ears to hear better than with one ear alone. Speech intelligibility is significantly improved due to dual ears when there are multiple interfering voices at different locations from the person, such as at a cocktail party [7]. This also enables sound localization (ability to identify the directional location of a sound source) [8].

\section{Article Objectives}

Inspired by the function of spatially separated sensory organs found in nature, this article explores the use of dual spatially separated sensors for enhanced estimation in modern engineering applications. The following three real-world applications are used to demonstrate the benefits of spatially separated redundant sensors in enabling enhanced estimation.

a) Adaptive parameter and state estimation in magnetic sensors: This application is used to demonstrate how spatially separated magnetic sensors can be used to estimate the position of a ferromagnetic object, even when the parameters of the magnetic field function are unknown and have to be adaptively estimated.

b) Estimation of an unknown disturbance input in an automotive suspension: This application demonstrates the real-time estimation of states and an unknown road roughness disturbance by using two spatially separated identical sensors in an automotive suspension.

c) Separation of inputs based on their direction of action in a digital stethoscope: This application is used to demonstrate how spatially separated redundant sensors can be exploited to separate two unknown disturbance inputs based on their direction of action.

This article presents both analytical components of observer design and experimental evaluation of the developed observer for each of the above applications. The common theme in all three applications is the use of dual sensors that measure the same type of variable, but are separated in spatial location. 


\section{State and Parameter Estimation in Systems with Nonlinear Measurement}

\section{Equations, with Applications to Magnetic Position Estimation}

Piston position measurement is required for many applications in a number of industrial domains. For example, in modern internal combustion engines with variable compression ratio, measuring the position of a piston inside the engine cylinder is important for real-time combustion control technologies [10, 11]. Another application is the measurement of a piston position in the hydraulic cylinders of an excavator for automatic excavation [12-14]. Piston position estimation is also required for many applications involving pneumatic actuators. One such application is web (continuous flexible materials such as cloth, foil and wire) handling where pneumatic actuators are used to control the position of guide rollers required for active control of tension in a web $[15,16]$.

Position transducers such as LVDTs (linear variable differential transformers) require a mechanical connection between the moving object and the sensor. Therefore, it is not possible to use these sensors in applications where the moving object is isolated in a harsh environment, for instance in a piston moving inside an engine cylinder or a piston in a hydraulic cylinder surrounded by high-pressure fluid. Laser and ultrasonic sensors do not require a mechanical connection to the moving object; however, they require a clear line of sight to the moving object. Therefore, their usage becomes difficult in situations where the moving object is isolated, for example to measure the internal piston position from outside the cylinder.

Magnetic sensors have also been used for the measurement of rotary and linear motions $[12,17,18]$. Previous position measurement systems based on embedded magnets have been developed only for applications with extremely small distances between the magnet and the sensor. For example, Hall-effect sensors typically work only at distances below $2 \mathrm{~cm}$ [19]. In order to measure the position of an object over a greater range, a continuous line of several magnets have to be embedded into the moving object $[12,18]$. This significantly increases installation burden and cost. Compared to the previous research in which the measurement range is very limited, this section shows how the nonlinear magnetic field model 
of an embedded magnet can be exploited to create a robust position sensor that works over much larger distances. In addition to improving the range, robustness is achieved by automatically determining the parameters of the magnetic field function using auto-calibration algorithms.

\section{The Analytical Observer Design Problem}

The process model of a piston-cylinder system is typically based on kinematics with linear process dynamics and a nonlinear measurement equation. The system dynamics are given by

$$
\dot{x}=A x+B u,
$$

where $x \in \mathbb{R}^{n \times 1}, A \in \mathbb{R}^{n \times n}, B \in \mathbb{R}^{n \times \ell}, u \in \mathbb{R}^{\ell \times 1}$.

The nonlinear measurement model is given by the output equation

$$
y=C x+h(x)
$$

where $y \in \mathbb{R}^{m \times 1}, C \in \mathbb{R}^{m \times n}$. See sidebar on "Observers for Nonlinear Systems with Output Nonlinear Functions," regarding the presence of the nonlinear function $h(x)$ in the measurement equation. It is assumed that the nonlinearity $h(x): \mathbb{R}^{n} \rightarrow \mathbb{R}^{m}$ is a vector of differentiable Lipschitz continuous functions whose Jacobian is bounded element-wise as follows

$$
K_{1}(i, j) \leq \frac{\partial h_{i}}{\partial x_{j}} \leq K_{2}(i, j), \quad i=1, \cdots, m ; \quad j=1, \cdots, n,
$$

where $K_{1}$ and $K_{2}$ are constant matrices of appropriate dimensions. Without loss of generality, it is assumed that

$$
K_{1}=0_{m \times n}
$$

It can be seen that if the lower Jacobian bound $K_{1}$ is non-zero, then the following new output nonlinearity function,

$$
\bar{h}(x)=-K_{1} x+h(x),
$$

has the following Jacobian bounds

$$
0 \leq \frac{\partial \bar{h}_{i}}{\partial x_{j}} \leq K_{2}(i, j)-K_{1}(i, j) .
$$


Thus the modified nonlinear function has a lower Jacobian bound of zero. The new output corresponding to this modified nonlinear function is

$$
y=\left(C+K_{1}\right) x+\bar{h}(x) .
$$

The nonlinear observer for this system will be designed based on the stability conditions for a Lur'e system shown in Figure 1.

The Lur'e system of Figure 1 is described by the equations

$$
\begin{aligned}
& \dot{x}=A x+B w, \\
& z=C x+D w, \\
& w=\Delta(z),
\end{aligned}
$$

where the nonlinear uncertain block $\Delta(\cdot)$ is an $\mathcal{L}_{2}$ operator. Assume that the relationship between $z$ and $w$ is constrained by a homogenous quadratic polynomial inequality given by

$$
\left[\begin{array}{l}
Z \\
w
\end{array}\right]^{T}\left[\begin{array}{cc}
Q & S \\
S^{T} & R
\end{array}\right]\left[\begin{array}{l}
Z \\
W
\end{array}\right] \leq 0
$$

where $Q, R$ and $S$ are real matrices with compatible dimensions. The following Lemma from a previous result in literature provides a sufficient condition for stability of a Lur'e system.

Lemma 1 (Acikmese and Corless, [20]):

The system given by equations (8) - (11) is globally asymptotically stable if there exists a symmetric matrix $P \in \mathbb{R}^{n \times n}$ such that

$$
P>0
$$

and

$$
\left[\begin{array}{cc}
A^{T} P+P A & P B \\
B^{T} P & 0
\end{array}\right]-[*]^{T}\left[\begin{array}{cc}
Q & S \\
S^{T} & R
\end{array}\right]\left[\begin{array}{cc}
C & D \\
0 & I
\end{array}\right] \prec 0
$$

where the symbols $>0$ and $\prec 0$ have been used to refer to a positive definite and negative definite matrix respectively. The observer for the nonlinear system of equations (1) and (2) is assumed to be

$$
\dot{\hat{x}}=A \hat{x}+B u+L_{1}\left(y-C \hat{x}-h\left(\hat{x}+L_{2}(y-C \hat{x}-h(\hat{x}))\right)\right),
$$

where the observer gains $L_{1}$ and $L_{2}$ are to be determined. 
It can then be shown that the observer error dynamics for the estimation error $e=x-\hat{x}$ is given by:

$$
\dot{e}=\left(A-L_{1} C\right) e-L_{1}\left[h(x)-h\left(\hat{x}+L_{2}(y-C \hat{x}-h(\hat{x}))\right)\right] .
$$

The second observer gain $L_{2}$ provides an additional degree of design freedom and has been used previously in other nonlinear observer design papers [21]. The error dynamics in (15) can then be represented as a Lur'e system, as shown in Figure 2 [21], [62].

The difference of nonlinear functions $\phi(t, e)$ in Figure 2 is

$$
\phi(t, e)=h(x)-h\left(\hat{x}+L_{2}(y-C \hat{x}-h(\hat{x}))\right) .
$$

It can then be shown [60] that with the lower Jacobian bound being zero, the error $e$ and the difference of nonlinear functions $\phi(t, e)$ satisfy

$$
[\phi(t, e)]^{T}\left[\phi(t, e)-K_{2}\left(I-L_{2} K\right) e\right] \leq 0,
$$

where $K \in \mathbb{R}^{m \times n}$ is time-varying, but also satisfies the element-wise constraints

$$
\begin{aligned}
& C(i, j) \leq K(i, j) \leq C(i, j)+K_{2}(i, j), \\
& \forall i=1, \ldots, m, j=1, \ldots, n
\end{aligned}
$$

The quadratic form of (17) is

$$
\left[\begin{array}{l}
e \\
\phi
\end{array}\right]^{T}\left[\begin{array}{cc}
I-L_{2} K & 0 \\
0 & I
\end{array}\right]^{T} M\left[\begin{array}{cc}
I-L_{2} K & 0 \\
0 & I
\end{array}\right]\left[\begin{array}{l}
e \\
\phi
\end{array}\right] \leq 0,
$$

where the multiplier matrix $M$ is defined as

$$
M=\left[\begin{array}{cc}
0 & -\frac{K_{2}^{T}}{2} \\
-\frac{K_{2}}{2} & I
\end{array}\right] .
$$

By applying Lemma 1 to the Lur'e system of Figure 2, linear matrix inequality (LMI) based observer design conditions can then be obtained for ensuring global asymptotic stability of the estimation error dynamics given by equation (15). These observer design conditions are presented as Theorem 1 below [53]. 
Theorem 1: The estimation error dynamics of equation (15) are asymptotically stable if there exist a symmetric matrix $P \in \mathbb{R}^{n \times n}$ and observer gains $L_{1} \in \mathbb{R}^{n \times m}$ and $L_{2} \in \mathbb{R}^{n \times m}$ such that the following semidefinite constraints are satisfied

$$
\begin{aligned}
& P>0 \text {, and } \\
& {\left[\begin{array}{cc}
\left(A-L_{1} C\right)^{T} P+P\left(A-L_{1} C\right) & -P L_{1} \\
-L_{1}^{T} P & 0
\end{array}\right]-[*]^{T} M\left[\begin{array}{cc}
I-L_{2} K & 0 \\
0 & I
\end{array}\right] \prec 0 .}
\end{aligned}
$$

This is an infinite-dimensional semidefinite constraint due to the time-varying (but bounded) matrix parameter $K$. However, the affine dependence on $K$ makes it only necessary to guarantee feasibility on all the $2^{m \times n}$ vertices of the polytope $\Xi$.

It is also possible to modify Theorem 1 as follows so that the estimation system satisfies a convergence rate of at least $\varepsilon$.

Corollary to Theorem 1: The existence of a matrix $P$ and two observer gains $L_{1}$ and $L_{2}$ such that the following semi-definite conditions are satisfied, implies exponential convergence with decay rate $\varepsilon>0$

$$
\begin{aligned}
& P>0 \text {, and } \\
& {\left[\begin{array}{cc}
\left(A-L_{1} C\right)^{T} P+P\left(A-L_{1} C\right)+2 \varepsilon P & -P L_{1} \\
-L_{1}^{T} P & 0
\end{array}\right]-[*]^{T} M\left[\begin{array}{cc}
I-L_{2} K & 0 \\
0 & I
\end{array}\right] \prec 0, \quad \text { for all } K \in \Xi .}
\end{aligned}
$$

\section{Application to Piston Position Estimation}

The above nonlinear measurement equation based nonlinear observer is applied to piston position estimation in a pneumatic actuator. Figure 3 shows a pneumatic actuator with a small (5 mm in diameter, $1 \mathrm{~mm}$ in thickness) magnet located on its piston. Magnetic sensors are located externally, co-axial with the cylinder. The position of the piston inside the pneumatic actuator can be estimated entirely from outside the cylinder non-intrusively.

It can be shown analytically [9] that the magnetic field measured by the two sensors in Figure 3 are given by 


$$
\begin{aligned}
& y_{1}=B_{1 x}=\frac{p}{(x+\sigma)^{3}}+q, \\
& y_{2}=B_{2 x}=\frac{p}{(x+\sigma+d)^{3}}+q,
\end{aligned}
$$

where the geometric parameters $\sigma$ and $d$ are shown in Figure 3. The redundancy created by using two magnetic sensors makes it possible to estimate both position and the unknown magnetic field parameters $p$ and $q$.

The state to be estimated is given by

$$
X=\left[\begin{array}{lllll}
x & v & a & p & q
\end{array}\right]^{T}
$$

and consists of position, velocity, acceleration, and the magnetic field parameters. The process dynamics are given by the kinematic model (28) while the parameters $p$ and $q$ are assumed to be constant and hence have the dynamics of equation (29),

$$
\begin{aligned}
& \frac{d}{d t}\left[\begin{array}{l}
x \\
v \\
a
\end{array}\right]=\left[\begin{array}{lll}
0 & 1 & 0 \\
0 & 0 & 1 \\
0 & 0 & 0
\end{array}\right]\left[\begin{array}{l}
x \\
v \\
a
\end{array}\right]+\left[\begin{array}{l}
0 \\
0 \\
1
\end{array}\right] J, \\
& \frac{d}{d t}\left[\begin{array}{l}
p \\
q
\end{array}\right]=\left[\begin{array}{ll}
0 & 0 \\
0 & 0
\end{array}\right]\left[\begin{array}{l}
p \\
q
\end{array}\right]
\end{aligned}
$$

where $J$ is jerk, and is assumed to be zero in this article. The measurement equations are given by equations (25) and (26). It is noted that with two spatially separated sensors, the linearized system corresponding to (25)-(29) is observable, whereas with just one sensor it would not be observable.

The observer in equation (14) is designed for the above system using the LMIs (23) and (24).

\section{Experimental Results}

The observer design from the previous section is experimentally implemented for position estimation of the piston in a pneumatic actuator. Figure 4 shows a photograph of the pneumatic actuator and experimental test configuration at the University of Minnesota. As shown, a set of two magnetic sensors on a circuit bread board are placed co-axial to the pneumatic actuator. A PIC-microcontroller based system is used for data acquisition. Since dual magnetic sensors with spatial separation are used (instead of a single magnetic sensor), the position of the piston can be estimated without requiring pre-calibration 
of the magnetic field function. Thus, the parameters of the magnetic field model are adaptively estimated in real-time using the spatially separated sensors. Sensor noise is not an issue in this application. Instead, the challenges in this application arise from unknown bias parameters in the sensor measurement.

Figure 5(a) shows the estimated piston position of the pneumatic actuator in which the piston moves over the full stroke of $50 \mathrm{~mm}$ and back to its original position. An LVDT is used as a reference sensor for comparison. The estimated position starts at an arbitrary initial value of $23 \mathrm{~mm}$ while the actual position measured by the LVDT is zero. The estimated position correctly converges to the actual position within a fraction of a second and tracks the actual position subsequently. The error in position is $23 \mathrm{~mm}$ in the beginning due to initial conditions but converges close to zero in less than 0.2 seconds, as seen in Figure 6(a). The estimated magnetic field parameters $p$ and $q$ converge even more quickly, as seen in Figures 5(b) and 5(c). The steady-state error in estimated position is seen to be less than $0.2 \mathrm{~mm}$, as shown in Figure 6(b).

As the results of this section demonstrate, the use of a redundant spatially separated sensor enables accurate position estimation for a nonlinear output equation system in spite of unknown magnetic field parameters.

\section{State and Disturbance Input Estimation in an Automotive Suspension}

Another application of the spatial redundant sensor configuration is the state and disturbance input estimation in an automotive suspension system. Figure 7 shows a schematic of a quarter car automotive suspension system. Such a model can be used to analyze vibrations of the automotive system due to road roughness $z_{r}$ at any one of the four wheels of the car [54]. The mass $m_{s}$ represents the vehicle body (or chassis) and is called the "sprung mass" while the mass $m_{u}$ represents the mass due to tire and axle [54] and is called the "unsprung mass." The sprung mass displacement is $z_{s}$, while the unsprung mass 
displacement is $z_{u}$. The automotive suspension itself consists of the suspension spring $k_{s}$ and the damper $b_{s}$. The tire stiffness is given by $k_{t}$.

If the suspension includes an active or semi-active vibration control element, the force provided by such an element is shown as $F_{a}$. This active suspension force $F_{a}$ can be a nonlinear function of the states and input (hydraulic actuator) or a bilinear function of the states and input (semi-active actuator) [54]. However, for the purposes of this article, vehicles with active or semi-active actuators, such as Formula race cars, are not considered. The estimation problem instead focuses on standard vehicles where no active control actuator is present $\left(F_{a}=0\right)$.

The governing differential equations of this system are given by [54]

$$
\begin{aligned}
& m_{s} \ddot{z}_{s}=-k_{s}\left(z_{s}-z_{u}\right)-b_{s}\left(\dot{z}_{s}-\dot{z}_{u}\right)+F_{a} \text {, and } \\
& m_{u} \ddot{z}_{u}=k_{s}\left(z_{s}-z_{u}\right)+b_{s}\left(\dot{z}_{s}-\dot{z}_{u}\right)-F_{a}-k_{t}\left(z_{u}-z_{r}\right) .
\end{aligned}
$$

The objective of the estimation problem is to estimate the following variables:
a) Tire deflection $z_{u}-z_{r}$
b) Suspension deflection $z_{s}-z_{u}$

Ideally, this estimation should be done using only inexpensive inertial sensors. Here we propose the use of two accelerometers - one each to measure the sprung mass acceleration $\ddot{z}_{S}$ and unsprung mass acceleration $\ddot{z}_{u}$. Although this system is observable using only a single sprung mass acceleration measurement output, the presence of the unknown disturbance $z_{r}$ requires additional consideration. By having two identical accelerometers spatially separated (one each on the sprung and unsprung mass), it is possible to estimate the desired state variables with complete decoupling from the unknown disturbance $z_{r}$. It is noted that the two accelerometers in this case are placed on existing separate masses, since it is not practically possible to introduce an additional redundant mass for the sake of a dual separated sensor on a real automotive suspension.

The incentive to estimate real-time tire deflection arises from a motivation to monitor road holding performance of the vehicle and to detect possible tire lift-off situations in real-time [24], [25]. Even 
without active suspensions, if potential tire-lift off can be predicted, the use of braking and deceleration of the vehicle (for instance, while cornering or doing a fast lane change) can prevent a vehicle from rollover.

The reason to estimate suspension deflection arises from a motivation to prevent bottoming out of suspension travel, since this can result in extremely bad ride quality and high jerk at the time of bottoming out. Bottoming out can also cause physical damage to the suspension system or vehicle body. Again, if this can be predicted by the estimation system, as simple a measure as braking to slow down the vehicle can prevent bottoming out.

The proposed accelerometers are inexpensive inertial sensors, with sprung mass acceleration already being measured on many cars. The desired state estimates can be decoupled from the unknown road input by using equation (31) which describes the unsprung mass dynamics. Since the unsprung mass acceleration $\ddot{z}_{u}$ is being measured, rewrite equation (31) so that the tire deflection is a function of the suspension deflection and velocity and the measured input $\ddot{z}_{u}$,

$$
z_{u}-z_{r}=\frac{1}{k_{t}}\left[b_{s}\left(\dot{z}_{s}-\dot{z}_{u}\right)+k_{s}\left(z_{s}-z_{u}\right)\right]-\frac{1}{k_{t}} m_{u} \ddot{z}_{u}
$$

This equation remains valid even for half car and full car suspension models. Next, using suspension deflection and relative suspension velocity as the reduced set of states to be estimated, the dynamic model for these two states can be represented using $\ddot{z}_{u}$ as a known input as follows

$$
\frac{d}{d t}\left[\begin{array}{l}
z_{s}-z_{u} \\
\dot{z}_{s}-\dot{z}_{u}
\end{array}\right]=\left[\begin{array}{cc}
0 & 1 \\
-\frac{k_{s}}{m_{s}} & -\frac{b_{s}}{m_{s}}
\end{array}\right]\left[\begin{array}{l}
z_{s}-z_{u} \\
\dot{z}_{s}-\dot{z}_{u}
\end{array}\right]+\left[\begin{array}{c}
0 \\
-1
\end{array}\right] \ddot{z}_{u}+\left[\begin{array}{c}
0 \\
\frac{1}{m_{s}}+\frac{1}{m_{u}}
\end{array}\right] u .
$$

The output is the sprung mass acceleration measurement, and hence the output equation is $y=C x$, with

$$
\begin{aligned}
& C=\left[\begin{array}{ll}
-\frac{k_{s}}{m_{s}} & -\frac{b_{s}}{m_{s}}
\end{array}\right], \\
& x=\left[\begin{array}{l}
z_{s}-z_{u} \\
\dot{z}_{s}-\dot{z}_{u}
\end{array}\right] .
\end{aligned}
$$

The system in equation (33) - (35) is observable and a Luenberger observer leads to asymptotically stable estimates for suspension deflection $z_{s}-z_{u}$ and velocity $\dot{z}_{s}-\dot{z}_{u}$. Subsequently, the tire deflection 
can be estimated using equation (32). Note that these deflections are obtained using only accelerometer measurements in a disturbance-affected system.

Simulation results on the above approach for estimating suspension deflection and tire deflection in a disturbance-affected quarter car suspension are shown in Figures 8 and 9. Figure 8 shows estimates and actual values of suspension and tire deflections when the road input disturbance is a $1 \mathrm{~Hz}$ sinusoid. After the initial error due to wrong initial conditions, the estimates converge rapidly (in about 1 sec) to the actual variables. Figure 9 shows the same type of data for a band-limited multi-frequency road input consisting of frequencies up to $10 \mathrm{~Hz}$. It can be seen that the estimates again converge rapidly and then track the actual values. Since tire deflection in particular is extremely difficult to measure on a real car, the observer developed herein using spatially separated sensors is of significant importance.

\section{Decoupling of Unknown Inputs, with Applications to}

\section{Auscultation in Medical Stethoscopes}

The third application presented in this article involves decoupling of two different unknown inputs in medical stethoscopes. Since the advent of the stethoscope in 1816, it has quickly risen to obtain status as the ubiquitous sign of a doctor. This is due largely to both the portability and the clinical relevance and versatility of the stethoscope as a physician's preliminary tool for assessment of a patient's circulatory and respiratory systems. The stethoscope amplifies chest sounds to a level of $60-70 \mathrm{~dB}$ at which point they can be heard by the physician. However, in emergency departments and ambulances, the existing environmental noise levels are high enough to make auscultation very difficult [46-49]. In the case of modern electronic stethoscopes, an additional potential noise source is found to be caused by a physician's handling of the stethoscope. This noise can be caused by finger/hand movement along the stethoscope chestpiece surface, accidental contact with the chestpiece, or muscle hand tremors. Since electronic stethoscopes have significantly larger chestpieces and metallic surface areas, they experience 
higher handling noise [26]. Mechanical isolation of the stethoscope’s transducer from these noise sources is possible, but this isolation is often achieved at the cost of a loss in sensitivity to patient chest sounds $[26]-[31]$.

Figure 10(a) shows a photograph of a modern electronic stethoscope and its internal components. Figure 10(b) shows a schematic model of the stethoscope. The model includes vibrational components as well as a piezoelectric element that converts chest vibrations to electrical signals [26].

As seen in Figure 10(b), there are two inputs that act on the system: the force due to handling of the stethoscope by the physician $F_{\text {medic }}$ and the force that the chest vibrations create on the diaphragm of the stethoscope $F_{\text {patient }}$.

The objective of this estimation problem is to estimate both the unknown inputs - the chest vibrations and the physician handling disturbance forces. It is impossible to eliminate the physician disturbance using only the single piezoelectric transducer. However, by using dual transducer measurements, the two unknown inputs can be estimated and the influence of the physician handling disturbances on the chest sound measurements can be removed.

\section{Review of Unknown Input Estimation Approaches}

The problem of unknown input estimation has long been investigated and typically arises in systems subject to disturbances, unmeasurable inputs, un-modeled dynamics, or in applications that require fault detection and isolation. For the linear time invariant system,

$$
\begin{gathered}
\dot{x}=A x+B d, \\
y=C x,
\end{gathered}
$$

where $x \in \mathbb{R}^{n}, d \in \mathbb{R}^{p}$, and $y \in \mathbb{R}^{p}$ and the system matrices $A, B$, and $C$ are known, constant, and of appropriate dimension, the objective is to estimate the unknown input, $d$, given the measured signal $y$.

Model inversion is one possible technique that has been established to generate a system mapping $R^{-1}: y \rightarrow u$ given an initial system map $R: u \rightarrow y$. Looking at the topic of functional 
reproducibility, Brockett and Mesarović [32] gave the first necessary and sufficient conditions for invertibility. An alternate test for invertibility has been presented by Sain [33], but these two criteria have since been found to be equivalent [34-36]. Brockett [38] provided an inversion algorithm for the linear time-invariant (LTI) single-input single-output (SISO) case. Dorato [40] derived a simplified criterion for invertibility and proposed a procedure for obtaining the inverse of a multiple-input multiple-output (MIMO) system. Additional research has been conducted to study the stability of such inverse systems. The conditions for the existence of a stable system inverse and its construction are given by Moylan [44]. These preliminary findings were primarily focused on the existence of such systems and their inherent system properties. However, they do not account for unknown initial conditions and do not estimate the states. Functional observers that provide disturbance-decoupled estimates of states or of portions of the state vector have been developed by other researchers [55]-[57].

Another approach for determining unknown inputs acting on a system is through the use of a socalled unknown input observer (UIO). Preliminary observer design in this area was in the interest of estimating the unknown state independent of the unmeasurable disturbances [37]. However, the unknown disturbance itself was not estimated. The first standard rank condition—the so called "Observer Matching Condition” was developed by Kudva [39] to determine when a reduced order UIO was possible. This requirement demonstrated that the necessary and sufficient condition for this class of observers to exist for a given system is the following simple condition

$$
\operatorname{rank}(C D)=\operatorname{rank}(D)=p,
$$

where $D$ is an added disturbance feedthrough term in equation (42), with $y=C x+D d$. Unfortunately, this necessary rank condition widely limits the applicability of rigorous UIOs to many real world systems, since the matrix $D$ can often be zero.

A large advance in UIO theory has been the development of observers for system which do not satisfy the observer matching condition. In general, these systems require the use of one or more output derivatives to successfully estimate the state and/or input. The work of Liu provides an approach for 
systems that violate the matching condition and have a relative degree equal to one [41]. However, this assumption on the relative degree is not valid for the stethoscope system which is explored here (and will generally not be valid for many applications).

More recently, Floquet and Zhu have presented methods for systems with a higher relative degree [42, 43]. The approaches of both authors rely on the use of high order sliding mode observers to estimate output derivatives. The methods which have been presented have complex design conditions and lack methods to add additional robustness considerations. What is presented here is a simpler method by which a linear observer can be designed which requires only the solution of a LMI and has a straightforward discrete time implementation.

\section{Analytical Preliminaries}

Without loss of generality, it can be assumed that the matrices $B$ and $C$ are full column and row rank respectively. In order to obtain an expression for the unknown input, $d$, we first differentiate the output from equation (42). After taking the output derivative and substituting for the state dynamics, equation (41), we obtain the following

$$
\dot{y}=C \dot{x}=C(A x+B d)
$$

Define the relative degree $r_{j}$ for the $j^{\text {th }}$ output as the number of times that this output needs to be differentiated for an input to appear, that is $C_{j} A^{i-1} B=0$ for all $1 \leq i \leq r_{j}-1$ and $C_{j} A^{r_{j}-1} B \neq 0$. Thus, after differentiating the $j^{\text {th }}$ output $r_{j}$ times, we obtain

$$
y_{j}^{\left(r_{j}\right)}=C_{j} A^{r_{j}} x+C_{j} A^{r_{j}-1} B d,
$$

where the superscript $\left(r_{j}\right)$ denotes the $r_{j}^{\text {th }}$ derivative of a variable. Without loss of generality, assume that $r_{1} \leq r_{2} \leq \cdots \leq r_{p}$. Then, the output derivatives can be combined in increasing order of relative degree in matrix form as follows, 


$$
\left[\begin{array}{c}
y_{1}^{\left(r_{1}\right)} \\
y_{2}^{\left(r_{2}\right)} \\
\vdots \\
y_{p}^{\left(r_{p}\right)}
\end{array}\right]=\left[\begin{array}{c}
C_{1} A^{r_{1}} \\
C_{2} A^{r_{2}} \\
\vdots \\
C_{p} A^{r_{p}}
\end{array}\right] x+\left[\begin{array}{c}
C_{1} A^{r_{1}-1} B \\
C_{2} A^{r_{2}-1} B \\
\vdots \\
C_{p} A^{r_{p}-1} B
\end{array}\right] d .
$$

This can be written in the following compact notation,

$$
\bar{y}=\bar{C} x+\bar{D} d,
$$

by defining $\bar{y} \in \mathbb{R}^{p}, \bar{C} \in \mathbb{R}^{p \times n}$, and $\bar{D} \in \mathbb{R}^{p \times p}$ as

$$
\bar{y}=\left[\begin{array}{c}
y_{1}^{\left(r_{1}\right)} \\
y_{2}^{\left(r_{2}\right)} \\
\vdots \\
y_{p}^{\left(r_{p}\right)}
\end{array}\right], \quad \bar{C}=\left[\begin{array}{c}
C_{1} A^{r_{1}} \\
C_{2} A^{r_{2}} \\
\vdots \\
C_{p} A^{r_{p}}
\end{array}\right], \quad \bar{D}=\left[\begin{array}{c}
C_{1} A^{r_{1}-1} B \\
C_{2} A^{r_{2}-1} B \\
\vdots \\
C_{p} A^{r_{p}-1} B
\end{array}\right]
$$

If the matrix $\bar{D}$ is invertible, then equation (42) can be solved to find the input, $d$, in terms of the output derivatives and states

$$
d=-\bar{D}^{-1} \bar{C} x+\bar{D}^{-1} \bar{y}
$$

Applying equation (44) to the original system equation, a new state equation without the unknown input can be obtained,

$$
\begin{aligned}
\dot{x} & =A x+B\left(-\bar{D}^{-1} \bar{C} x+\bar{D}^{-1} \bar{y}\right) \\
& =\left(A-B \bar{D}^{-1} \bar{C}\right) x+B \bar{D}^{-1} \bar{y}
\end{aligned}
$$

If we define the matrices,

$$
\begin{array}{ll}
A_{b}=A-B \bar{D}^{-1} \bar{C}, & B_{b}=B \bar{D}^{-1}, \\
C_{b}=-\bar{D}^{-1} \bar{C}, & D_{b}=\bar{D}^{-1},
\end{array}
$$

the new dynamic equations relating the original system output to an estimate of the input is given by,

$$
\begin{aligned}
& \dot{x}=A_{b} x+B_{b} \bar{y}, \\
& d=C_{b} x+D_{b} \bar{y} .
\end{aligned}
$$

The resulting system requires $r_{j}$ derivatives of the measured output signal $y_{j}$ to estimate the original system input(s). 


\section{Unknown Input Observer}

As an alternate method to estimate the unknown input of a system, it is possible to design an unknown input and state observer that does not rely on the construction of an explicit inverse dynamic model. The following result presents an observer and LMIs that constitute design constraints on the observer gains for asymptotic state and input estimation.

First, for a system where each output has a relative degree $r_{j}$ as previously defined, define the structures of the matrices, $\mathcal{G} \in \mathbb{R}^{p \times(\tilde{r}+q)}, \mathcal{H} \in \mathbb{R}^{p \times q}, \tilde{\mathcal{C}} \in \mathbb{R}^{(\tilde{r}+q) \times n}$, and $\widetilde{\mathcal{D}} \in \mathbb{R}^{q \times p}$ as follows,

$$
\begin{array}{rlll}
\mathcal{G} & =\left[\begin{array}{llll}
G_{1} & G_{2} & \cdots & G_{q}
\end{array}\right], & \mathcal{H} & =\left[\begin{array}{llll}
H_{1} & H_{2} & \cdots & H_{q}
\end{array}\right], \\
\tilde{\mathcal{C}}=\left[\begin{array}{c}
\tilde{C}_{1} \\
\tilde{C}_{2} \\
\vdots \\
\tilde{C}_{q}
\end{array}\right], & \widetilde{\mathcal{D}}=\left[\begin{array}{c}
\widetilde{D}_{1} \\
\widetilde{D}_{2} \\
\vdots \\
\widetilde{D}_{q}
\end{array}\right],
\end{array}
$$

where

$$
\tilde{r}=\sum_{i=1}^{q} r_{i}
$$

and the sub-matrices $G_{i} \in \mathbb{R}^{p \times\left(r_{i}+1\right)}, H_{i} \in \mathbb{R}^{p \times 1}, \tilde{C}_{i} \in \mathbb{R}^{\left(r_{i}+1\right) \times n}$, and $\widetilde{D}_{i} \in \mathbb{R}^{1 \times p} \forall i \in\{1,2, \ldots, q\}$ are defined as

$$
\begin{array}{rlll}
G_{i} & =\left[\begin{array}{llll}
G_{i, 0} & G_{i, 1} & \cdots & G_{i, r_{i}}
\end{array}\right], & H_{i}=G_{i, r_{i}}, \\
\tilde{C}_{i}=\left[\begin{array}{c}
C_{i} \\
C_{i} A \\
\vdots \\
C_{i} A^{r_{i}}
\end{array}\right], & & &
\end{array}
$$

Then, define the observer update laws as follows

$$
\begin{aligned}
& \dot{\hat{x}}=(A-L C) \hat{x}+B \hat{d}+L y, \\
& \dot{\hat{d}}=-\mathcal{G} \tilde{\mathcal{C}} \hat{x}-\mathcal{H} \widetilde{\mathcal{D}} \hat{d}+\mathcal{G} \tilde{y},
\end{aligned}
$$

where $L \in \mathbb{R}^{n \times q}$ is the observer gain to be determined and the output derivative vector, $\tilde{y} \in \mathbb{R}^{\tilde{r}+q}$, is defined as 


$$
\tilde{y}=\left[\begin{array}{c}
\tilde{y}_{1} \\
\tilde{y}_{2} \\
\vdots \\
\tilde{y}_{q}
\end{array}\right],
$$

with $\tilde{y}_{i} \in \mathbb{R}^{r_{i}+1}$,

$$
\tilde{y}_{i}=\left[\begin{array}{c}
y_{i} \\
\dot{y}_{i} \\
\vdots \\
y_{i}^{\left(r_{i}\right)}
\end{array}\right] .
$$

Note that equation (53) for the disturbance estimates utilizes all available feedback, namely the state estimate $\hat{x}$, the disturbance estimate $\hat{d}$ and the derivatives of the measurement $\tilde{y}$. Defining the state and input estimate errors as

$$
e_{x}=x-\hat{x}, \quad e_{d}=d-\hat{d},
$$

the observer state estimate can be rewritten as

$$
\dot{\hat{x}}=A \hat{x}+B \hat{d}+L C e_{x} .
$$

Similarly, after some manipulation, the unknown input estimate can be written as

$$
\dot{\hat{d}}=\mathcal{G} \tilde{\mathcal{C}} e_{x}+\mathcal{H} \widetilde{\mathcal{D}} e_{d}
$$

Theorem 2. If there exist observer gain matrices $L$ and $\mathcal{G}$ and two symmetric positive-definite (SPD) matrices $P_{x}, P_{d}$, such that

$$
Q=\left[\begin{array}{cc}
-P_{x}(A-L C)-(A-L C)^{\mathrm{T}} P_{x} & -P_{x} B+\tilde{\mathcal{C}}^{\mathrm{T}} \mathcal{G}^{\mathrm{T}} P_{d} \\
-B^{\mathrm{T}} P_{x}+P_{d} \mathcal{G} \tilde{\mathcal{C}} & P_{d} \mathcal{H} \widetilde{\mathcal{D}}+\widetilde{\mathcal{D}}^{\mathrm{T}} \mathcal{H}^{\mathrm{T}} P_{d}
\end{array}\right]<0,
$$

then the observer given by

$$
\begin{aligned}
& \dot{\hat{x}}=(A-L C) \hat{x}+B \hat{d}+L y, \\
& \dot{\hat{d}}=-\mathcal{G} \tilde{\mathcal{C}} \hat{x}-\mathcal{H} \widetilde{\mathcal{D}} \hat{d}+\mathcal{G} \tilde{y},
\end{aligned}
$$

can be used to asymptotically estimate both the state and the unknown input.

Proof: The theorem is proved using the Lyapunov function candidate

$$
V=e_{x}^{\mathrm{T}} P_{x} e_{x}+e_{d}^{\mathrm{T}} P_{d} e_{d}
$$


It can be shown that if there exists symmetric positive-definite matrices $P_{x}, P_{d}$, and $Q$ that satisfy equation (59), then $V$ is positive definite and $\dot{V}$ is negative definite on the entire space $\mathbb{R}^{n+p}$ [26]. Additionally, since $V$ is radially unbounded, it is possible to conclude that $e_{x}=0$ and $e_{d}=0$ is a globally asymptotically stable equilibrium point ([62], Theorem 4.2). Thus, the given observer can guarantee that both the state and unknown input can be asymptotically tracked. It should be noted that although the disturbance was assumed to be an unknown constant, in practice a time varying disturbance can also be estimated in real-time if the sampling frequency is adequately fast and the observer dynamics are significantly faster than the bandwidth of the disturbance.

\section{Stethoscope Input Estimation}

Figure 11 shows a schematic of a modified stethoscope at the University of Minnesota with dual piezoelectric transducers separated spatially by a spring in between the two transducers. This stethoscope can be constructed by modification of a standard 3M Littman 3200 electronic stethoscope [45]. By using dual transducer measurements, the two unknown inputs can be estimated and the influence of the physician handling disturbances on the chest sound measurements can be removed. Figure 12 shows the modified schematic for the dynamic model of the dual piezo transducer configuration in the stethoscope.

In state-space form, the physical system’s dynamic model is given as follows,

$$
\begin{gathered}
\dot{x}_{\mathrm{d}}=A_{d} x_{d}+B_{d} d, \\
y_{d}=C_{d} x_{d},
\end{gathered}
$$

where $x_{d} \in \mathbb{R}^{8}$ is the state and $d \in \mathbb{R}^{2}$ is the unknown input,

$$
d=\left[\begin{array}{ll}
F_{p} & F_{m}
\end{array}\right]^{\mathrm{T}} .
$$

The specific numerical system matrices $A_{d} \in \mathbb{R}^{8 \times 8}, B_{d} \in \mathbb{R}^{8 \times 2}$, and $C_{d} \in \mathbb{R}^{2 \times 8}$ can be found in the doctoral thesis [26]. This model can be used to relate the vibrational inputs to pressure on the piezo 
sensing elements [50]. However, a conversion is required to relate these forces to output voltages. The state-space representation of the differential equations relating pressure and voltage for piezo $i$ can be written as follows

$$
\begin{gathered}
\dot{x}_{p_{i}}=A_{p_{i}} x_{p_{i}}+B_{p_{i}} u_{p_{i}}, \\
y_{p_{i}}=C_{p_{i}} x_{p_{i}},
\end{gathered}
$$

where $\mathrm{x}_{p_{i}} \in \mathbb{R}^{3}$ and the system matrices $A_{p_{i}} \in \mathbb{R}^{3 \times 3}, \mathrm{~B}_{p_{i}} \in \mathbb{R}^{3 \times 1}$, and $C_{p} \in \mathbb{R}^{1 \times 3}$ are given in [26].

The output of each piezo sensor is quite small. In order to amplify and improve the signal quality, a small preamplifier circuit is used for each piezo. The state-space model for amplifier $i$ is given by the following

$$
\begin{gathered}
\dot{x}_{a_{i}}=A_{a_{i}} x_{a_{i}}+B_{a_{i}} u_{a_{i}}, \\
y_{a_{i}}=C_{a_{i}} x_{a_{i}},
\end{gathered}
$$

where $\mathrm{x}_{a_{i}} \in \mathbb{R}^{5}$ and the system matrices are given in [26]. For ease of development, the two preamplifier models will be stacked to create a decoupled two input and two output system, $G_{a}$, given by $\left(A_{a}, B_{a}, C_{a}\right)$.

$$
A_{a}=\left[\begin{array}{cc}
A_{a_{1}} & 0 \\
0 & A_{a_{1}}
\end{array}\right], \quad B_{a}=\left[\begin{array}{cc}
B_{a_{1}} & 0 \\
0 & B_{a_{1}}
\end{array}\right] \quad, C_{a}=\left[\begin{array}{cc}
C_{a_{1}} & 0 \\
0 & C_{a_{1}}
\end{array}\right]
$$

Similarly, the two piezo models have been stacked and the resulting system, $G_{p}$, is given by $\left(A_{p}, B_{p}, C_{p}\right)$.

$$
A_{p}=\left[\begin{array}{cc}
A_{p_{1}} & 0 \\
0 & A_{p_{1}}
\end{array}\right], \quad B_{p}=\left[\begin{array}{cc}
B_{p_{1}} & 0 \\
0 & B_{p_{1}}
\end{array}\right], \quad C_{p}=\left[\begin{array}{cc}
C_{p_{1}} & 0 \\
0 & C_{p_{1}}
\end{array}\right] .
$$

Schematically, the interconnection of the sub-systems can be seen in Figure 13. The following change of notation is introduced for the complete system model: $u_{1} \triangleq F_{p}, u_{2} \triangleq F_{m}, y_{1} \triangleq y_{a_{1}}$, and $y_{2} \triangleq y_{a_{2}}$.

\section{Stethoscope Observer Design}

It is possible to construct a full system observer for the combined model. However, here we have chosen to design an unknown input observer for each cascaded subsystem. This minimizes the number of output derivatives required for each estimator (and thus reduces the effect of measurement noise). 
The dynamic model, $G_{d}$, has well defined relative degree equal to one for each output $\left(r_{d_{1}}=1, r_{d_{2}}=\right.$ 1). The piezo subsystems each have a relative degree of three $\left(r_{p_{1}}=3, r_{p_{2}}=3\right)$, and each preamplifier subsystem has a relative degree of two $\left(r_{a_{1}}=2, r_{a_{2}}=2\right)$.

The inversion based observer for this system can be given by

$$
\begin{aligned}
& \dot{\hat{x}}_{a}=\left(A_{b, a}-L_{a} C_{a}\right) \hat{x}_{a}+B_{b, a} \bar{y}_{a}+L_{a} y_{a}, \\
& \dot{\hat{x}}_{p}=\left(A_{b, p}-L_{p} C_{p}\right) \hat{x}_{p}+B_{b, p} \hat{\bar{y}}_{p}+L_{p} \hat{y}_{p}, \\
& \dot{\hat{x}}_{d}=\left(A_{b, d}-L_{d} C_{d}\right) \hat{x}_{d}+B_{b, d} \hat{\bar{y}}_{d}+L_{d} \hat{y}_{d},
\end{aligned}
$$

where the observer gains $L_{a} \in \mathbb{R}^{8 \times 2}, L_{p} \in \mathbb{R}^{6 \times 2}$, and $L_{d} \in \mathbb{R}^{10 \times 2}$ were selected as

$$
\begin{aligned}
& L_{d}= \\
& {\left[\begin{array}{cccccccc}
-3.39 \mathrm{e}^{-3} & -4.30 \mathrm{e}^{-3} & -1.04 \mathrm{e}^{-2} & -1.73 \mathrm{e}^{-2} & -3.99 \mathrm{e}^{-4} & -5.27 \mathrm{e}^{-3} & 5.76 \mathrm{e}^{-3} & -1.74 \mathrm{e}^{-3} \\
-1.77 \mathrm{e}^{-3} & -1.52 \mathrm{e}^{-3} & 8.06 \mathrm{e}^{-4} & 5.42 \mathrm{e}^{-4} & 2.68 \mathrm{e}^{-4} & -4.26 \mathrm{e}^{-4} & 3.60 \mathrm{e}^{-5} & 2.21 \mathrm{e}^{-4}
\end{array}\right]^{\mathrm{T}},} \\
& L_{p}=\left[\begin{array}{cccccc}
516.16 & 713052.69 & 2797.54 & 0 & 0 & 0 \\
0 & 0 & 0 & 240.93 & 1078955.40 & 3684.15
\end{array}\right]^{\mathrm{T}}, \\
& L_{a}=\left[\begin{array}{cc}
43.08 & 0 \\
151.58 & 0 \\
280.68 & 0 \\
2157.8 & 0 \\
709.71 & 0 \\
0 & 1.5636 \\
0 & -6.0234 \\
0 & 37.024 \\
0 & 443.2 \\
0 & 348.38
\end{array}\right] \text {, }
\end{aligned}
$$

by solving the linear matrix inequality given by condition that a positive definite $P$ and $Q$ must exist where $Q$ is defined by equation (59).

The state matrix $\left(A_{b, a}, A_{b, p}\right.$, and , $\left.A_{b, d}\right)$ and input matrix $\left(B_{b, a}, B_{b, p}\right.$, and , $\left.B_{b, d}\right)$ for each observer were obtained by equation (46) based on the original dynamic model for each subsystem. There are two inputs to the first observer given by equation (72). As the first stage in the series of three observers, the feedback term $y_{a}$ for this observer is the measured signal from the piezo, and the inverted system input $\bar{y}_{a}$ is a 
vector of the measurement derivatives as defined by equation (43). For each subsequent observer in the series, the inputs are similarly defined.

Due to the cascaded observer design selected, all systems after the first observer rely on the use of output estimates (the result of the prior observer's estimate) and not a direct measurement. In the steady state, the internal stability of each observer guarantees that the cascaded observer will converge.

Similarly, a non-inversion based observer can be design for the stethoscope system. The state estimates are given by the following

$$
\begin{aligned}
& \dot{\hat{x}}_{a}=\left(A_{a}-L_{a} C_{a}\right) \hat{x}_{a}+B_{a} \hat{d}_{a}+L_{a} y_{a}, \\
& \dot{\hat{x}}_{p}=\left(A_{p}-L_{p} C_{p}\right) \hat{x}_{p}+B_{p} \hat{d}_{p}+L_{p} \hat{y}_{p}, \\
& \dot{\hat{x}}_{d}=\left(A_{d}-L_{d} C_{d}\right) \hat{x}_{d}+B_{d} \hat{d}_{d}+L_{d} \hat{y}_{d} .
\end{aligned}
$$

The update laws for the unknown input estimates for each stage of the observer are given by

$$
\begin{aligned}
& \dot{\hat{d}}_{a}=-\mathcal{G}_{a} \tilde{\mathcal{C}}_{a} \hat{x}_{a}-\mathcal{H}_{a} \widetilde{\mathcal{D}}_{a} \hat{d}_{a}+\mathcal{G}_{a} \tilde{\mathcal{Y}}_{a}, \\
& \dot{\hat{d}}_{p}=-\mathcal{G}_{p} \tilde{\mathcal{C}}_{p} \hat{x}_{p}-\mathcal{H}_{p} \widetilde{\mathcal{D}}_{p} \hat{d}_{p}+\mathcal{G}_{p} \tilde{\mathcal{Y}}_{p}, \\
& \dot{\hat{d}}_{d}=-\mathcal{G}_{d} \tilde{\mathcal{C}}_{d} \hat{x}_{d}-\mathcal{H}_{d} \widetilde{\mathcal{D}}_{d} \hat{d}_{d}+\mathcal{G}_{d} \tilde{\mathcal{Y}}_{d}
\end{aligned}
$$

The state observer gains $L_{a} \in \mathbb{R}^{8 \times 2}, L_{p} \in \mathbb{R}^{6 \times 2}$, and $L_{d} \in \mathbb{R}^{10 \times 2}$ and the disturbance observer gains $\mathcal{G}_{a} \in$ $\mathbb{R}^{2 \times 4}, \mathcal{G}_{p} \in \mathbb{R}^{2 \times 4}, \mathcal{G}_{d} \in \mathbb{R}^{2 \times 6}, \mathcal{H}_{a} \in \mathbb{R}^{2 \times 2}$, and $\mathcal{H}_{p} \in \mathbb{R}^{2 \times 2}$ were obtained by solving the linear matrix inequality given by condition that a positive definite $P$ and $Q$ must exist where $Q$ is defined by equation (59).

Similar to the inversion based observer, there are two inputs to each stage of the observer. The input to the amplifier system observer, $y_{a}$, is the measured signal from the piezo and the estimated input given defied by equation (79). The input to the unknown input observer for this system $\tilde{y}_{a}$ is a vector of the measurement derivatives as defined by equations (54) and (55). For each subsequent observer in the series, the inputs are similarly defined. Once again, the measurements used by the second and third observer are given by the estimated output from the observer which precedes it. 


\section{Experimental Results}

In order to experimentally verify the proposed design and algorithm on the physical system, a prototype of the proposed dual-piezo assembly was constructed and tested. Experimental testing was accomplished by stimulating the dual-piezo assembly with vibrational noise from both potential noise sources. For the generation of patient noise, a vibration shaker was used to generate a vibrational noise signal representative of a standard heartbeat. During each test, the housing for the first piezo assembly was bonded to the shaker via a foam disk with double-sided adhesive tape. The second unknown input, a disturbance due to physician handling noise, could be created by rubbing and tapping on the top surface of the second (upper) piezo housing. Although a known reference disturbance signal was not used for this noise source, knowledge of the desired chest sound signal allows for a sufficient assessment of the algorithm’s performance.

In order to monitor the actual input acceleration experienced by the sensor assembly, a single axis accelerometer was rigidly attached to the shaker head. A National Instruments CompactRio chassis (NI cRIO-9074) with an analog input (NI 9205) and an analog output (NI 9264) module has been used to generate and acquire the test signals [61]. A constant sampling rate of $25 \mathrm{kHz}$ has been used for all results presented.

Results using an initial white noise only patient input with no added disturbance signal are presented herein. The following measurements from each piezo sensor were obtained (see Figure 14).

Since only a single input was present, after processing the data through the designed observer, the signal energy should all be attributed to a single input. More specifically, all the input should be identified as having originated from input $1\left(u_{1}\right)$. As shown in Figure 16, in general this is confirmed in the processed data. 
For frequency components below approximately $800 \mathrm{~Hz}$, the signal estimate agrees well with the original input signal. In this same frequency range, the second input estimate has been reduced greatly. However, in both datasets it can be seen that considerable high frequency components exist which diverge from the desired signal (see Figure 15). These errors are once again due to the numerical derivative approximation and the given choice of observer gains. Given that much of the estimate error lies above the frequency range of interest, it can be removed with filtering. After appropriate low-pass filtering, the time domain signal estimates shown in Figure 16 are obtained.

The estimate of the first input agrees well with the actual input signal used for the experiment. Additionally, as desired, the amplitude of the second input estimate is considerably smaller.

In summary, existing approaches to minimize the effect of disturbances caused by a physician during auscultation have been focused on passive improvements to the design. These methods attempt to isolate the transducer from such input signals and dissipate their energy prior to reaching the measurement sensor. Depending on the nature of the design, this isolation may not achieve sufficient noise reduction without compromising the device's sensitivity to patient signals. Using spatially separated sensors and an unknown input observer design based on a model of how these interference signals reach each sensor, their effects can be removed digitally.

An unknown input observer design has been presented, which does not rely explicitly on the system inverse model, to estimate both the states and the two unknown inputs. Its performance has been demonstrated on a simplified laboratory system using a dual piezo sensor assembly. The use of numerical differentiation required within each algorithm degrades the estimate performance in the high frequency range, but low frequency estimates demonstrate a high level of performance.

Using the developed approach, it is feasible that the unwanted effects of disturbances caused by a physician can be reduced while providing a high quality estimate of the original auscultation signal. This technology can improve the stethoscope's performance and generally improve the quality of patient examination possible in a wide variety of environments. 


\section{Conclusions}

This work on the use of dual identical but spatially separated sensors was inspired by the dual sensing organs in humans and other creatures in nature. The article showed how the use of dual sensors could be used to compensate for unknown parameters and unknown disturbance inputs, while estimating states in linear and nonlinear dynamic systems. Applications of the dual sensor methodology were discussed for

a) An industrial pneumatic actuator system in which magnetic sensors were used for position estimation without requiring pre-calibration of magnetic field parameters,

b) An automotive systems in which dual accelerometers were used to estimate states and an unknown tire deflection input, and

c) An electronic stethoscope in which unknown physician created handling noise was estimated and removed from chest sounds to enable medical auscultation.

A building window with a transparent acoustic speaker in which dual microphones were used to separate internal and external noise was also discussed briefly (See sidebar on "Application to Separation of Directional Noise Components in Acoustics”).

Both analytical observer design results and experimental implementation results for these applications were presented.

\section{References}

[1] I.P. Howard and B.J. Rogers, Binocular Vision and Stereopsis, Oxford University Press, New York, 1995.

[2] R.K. Jones and D.N. Lee, "Why two eyes are better than one: The two views of binocular vision," Journal of Experimental Psychology: Human Perception and Performance, Vol. 7, pp. 30-40, 1981. 
[3] K.J. Ciuffreda, "Why two eyes?” available at http://www.svs-greensboro.com/why2eyes.php, Last accessed on September 5, 2015.

[4] C.M. van Mierlo, E. Brenner and J.B.J. Smeets, "Better performance with two eyes than with one in stereo-blind subjects’ judgements of motion in depth,” Vision Research, Vol. 51, pp. 1249-1253, 2011.

[5] M.A. Changizi and S. Shimojo, "X-ray vision and the evolution of forward facing eyes," Journal of Theoretical Biology, Vol. 254, No. 4, pp. 756-767, 2008.

[6] J. Frasnelli, “Why do we have 2 nostrils?” Odor Management Blog, Available at http://blog.odotech.com/2-nostrils-special-guest-author-edition, Last accessed on Sep 5, 2015.

[7] M.L. Hawley and R.Y. Litovsky, "The benefit of binaural hearing in a cocktail party: Effect of location and type of interferer,” Journal of the Acoustical Society of America, Vol. 115, No. 2, February 2004.

[8] J.W.H. Schnupp and C.E. Carr, "On hearing with more than one ear: lessons from evolution," Nature Neuroscience, Vol. 12, No. 6, pp. 692-697, 2009.

[9] S. Taghvaeeyan and R. Rajamani, "Magnetic sensor based large distance position estimation with disturbance compensation," IEEE Sensors J., vol. 15, no. 8, pp. 4249-4258, August, 2015.

[10] T. A. Johansen, O. Egeland, E. A. Johannessen and R. Kvamsdal, "Free-piston diesel engine timing and control-toward electronic cam-and crankshaft," Control Systems Technology, IEEE Transactions On 10(2), pp. 177-190. 2002.

[11] K. Li, A. Sadighi and Z. Sun, “Active motion control of a hydraulic free piston engine,” Mechatronics, IEEE/ASME Transactions On 19(4), pp. 1148-1159. 2014.

[12] S. Y. Yang, M. C. Lee, M. H. Lee and S. Arimoto, "Measuring system for development of strokesensing cylinder for automatic excavator,” Industrial Electronics, IEEE Transactions on 45(3), pp. 376-384. 1998. 
[13] H. Shao, H. Yamamoto, Y. Sakaida, T. Yamaguchi, Y. Yanagisawa and A. Nozue, “Automatic excavation planning of hydraulic excavator,” Presented at Proceedings of the First International Conference on Intelligent Robotics and Applications: Part II. 2008.

[14] H. Yamamoto, M. Moteki, H. Shao, T. Ootuki, H. Kanazawa and Y. Tanaka, "Basic technology toward autonomous hydraulic excavator," Presented at 26th International Symposium on Automation and Robotics in Construction (ISARC 2009). 2009.

[15] P. R. Pagilla, R. V. Dwivedula, Y. Zhu and L. P. Perera, "Periodic tension disturbance attenuation in web process lines using active dancers,” Journal of Dynamic Systems, Measurement, and Control 125(3), pp. 361-371. 2003.

[16] V. Gassmann, D. Knittel, P. R. Pagilla and M. Bueno, “Fixed-order tension control in the unwinding section of a web handling system using a pendulum dancer,” Control Systems Technology, IEEE Transactions On 20(1), pp. 173-180. 2012.

[17] P. Campbell, “Magnetoresistive sensors for rotary position encoders,” Magnetics, IEEE Transactions On 26(5), pp. 2029-2031. 1990.

[18] B. Hebert, M. Brule and L. Dessaint, “A high efficiency interface for a biphase incremental encoder with error detection [servomotor control],” Industrial Electronics, IEEE Transactions On 40(1), pp. 155-156. 1993.

[19] E. Ramsden, Hall-Effect Sensors: Theory and Application. Amsterdam, The Netherlands: Elsevier, 2011.

[20] B. Acikmese and M. Corless, “Observers for Systems with Nonlinearities Satisfying Incremental Quadratic Constraints,” Automatica, Vol. 47, No. 7, pp. 1339-1348, 2011.

[21] M. Arcak and P. Kokotovic, "Nonlinear Observers: A Circle Criterion Design and Robustness Analysis,” Automatica, Vol. 37, No. 12, pp. 1923-1930, 2001. 
[22] A. Zemouche, M. Boutayeb and G. Iulia Bara, "Observers for a Class of Lipschitz Systems with Extension to $H_{\infty}$ Performance Analysis,” Systems and Control Analysis, Vol. 57, No. 1, pp. 18-27, 2008.

[23] A. Zemouche and M. Boutayeb, "On LMI Conditions to design Observers for Lipschitz Nonlinear Systems,” Automatica, Vol. 49, No. 2, pp. 585-591, 2013.

[24] J.K. Hedrick, R. Rajamani and K. Yi, “Observer Design for Electronic Suspension Applications,” Vehicle System Dynamics, Vol. 23, No. 1, pp. 413-440, 1994.

[25] K. Yi and J.K. Hedrick, “Dynamic Tire Force Control by Semi-Active Suspensions,” ASME Journal of Dynamic Systems, Measurement and Control, Vol. 115, No. 3, pp. 465-474, Sep 1, 1993.

[26] Garrett D. Nelson, “Stethoscope Design for Auscultation in High Noise Environments,” Ph.D. Thesis, Department of Mechanical Engineering, University of Minnesota, Oct 31, 2015.

[27] G. Zenk, "Stethoscopic Detection of Lung Sounds in High Noise Environments,” West Lafayette, IN: Purdue University, 1994.

[28] H. Greenberger, "Vibration isolation mount for a stethoscope chestpiece, and methods of using same". U.S. Patent 5,883,339, 16 Mar. 1999.

[29] F. Bilan, "Floating Ballast Mass Active Stethoscope or Sound Pickup Device". U.S. Patent 2009/0211838, 27 Feb 2008.

[30] R. Cusson, "Resonant Chamber Sound Pick-Up". U.S. Patent 6587564, 1 Jul 2003.

[31] T. Drummond, H. Carim and C. Oster, "Stethoscope with frictional noise reduction". U.S. Patent 7,806,226 B2, 5 Oct. 2010.

[32] R. W. Brockett and M. D. Mesarovic, "The reproducibility of multivariable systems," J. Math. Anal. Applicat., vol. 11, no. 1, pp. 548-563, 1965.

[33] M. K. Sain and J. L. Massey, "Invertibility of linear time-invariant dynamical systems," IEEE Trans. Automat. Control, vol. 14, no. 2, pp. 141-149, 1969. 
[34] S. P. Singh, "A note on inversion of linear systems," IEEE Trans. Automat. Control, vol. 15, no. 4, pp. 492-493, 1970.

[35] S. P. Panda, L. M. Silverman, M. K. Sain and J. L. Massey, "Comments on "Inversion of multivariable linear systems"," IEEE Trans. Automat. Control, vol. 15, no. 4, pp. 489-491, 1970.

[36] A. S. Willsky, "On the invertibility of linear systems," IEEE Trans. Automat. Control, vol. 19, no. 3, pp. 272-274, 1974.

[37] S. H. Wang and E. J. Davison, "A new invertibility criterion for linear multivariable systems," IEEE Trans. Automat. Control, vol. 18, no. 5, pp. 538-539, 1973.

[38] R. W. Brockett, "Poles, zeros, and feedback: State space interpretation," IEEE Trans. Automat. Control, vol. 10, no. 2, pp. 129-135, 1965.

[39] P. Kudva, N. Viswanadham and A. Ramakrishna, "Observers for linear systems with unknown inputs," IEEE Trans. Automat. Control, vol. 25, no. 1, pp. 113-115, 1980.

[40] P. Dorato, "On the inverse of linear dynamical systems," IEEE Trans. Syst. Sci. Cybern., vol. 5, no. 1, pp. 43-48, 1969.

[41] C.-S. Liu and H. Peng, "Inverse-dynamics based state and disturbance observers for linear timeinvariant systems," J. Dynamic Syst. Meas. Control, vol. 124, no. 3, pp. 375-381, 2002.

[42] T. Floquet, C. Edwards and S. K. Spurgeon, "On sliding mode observers for systems with unknown inputs," Int. J. Adapt. Control Signal Process., vol. 21, no. 8-9, pp. 638-656, 2007.

[43] F. Zhu, "State estimation and unknown input reconstruction via both reduced-order and high-order sliding mode observers," J. Process. Control, vol. 22, no. 1, pp. 296-302, 2012.

[44] P. J. Moylan, "Stable inversion of linear systems," IEEE Trans. Automat. Control, vol. 22, no. 1, pp. 74-78, 1977.

[45] 3M, "3M Littmann Stethoscopes," 2013. [Online]. Available: http://www.littmann.com/wps/portal/3M/en_US/3M-Littmann/stethoscope/stethoscope- 
catalog/catalog/ /3M-Littmann-Electronic-Stethoscope-Model-Black-Tube-27-inch3200BK27?N=5932256+4294958300\&rt=d. [Accessed 23 May 2013].

[46] L. Zun and L. Downey, "The Effect of Noise in the Emergency Department," Academic Emergency Medicine, vol. 12, no. 7, pp. 663-666, 2005.

[47] D. Groom, "The Effect of Bacground Noise on Cardiac Auscultation," American Heart Journal, vol. 52, no. 5, pp. 781-790, 1956.

[48] H. Greenberger, "Vibration Isolation Mount for a Stethoscope Chestpiece, and Methods of Using Same". U.S. Patent 5883339, 16 Mar 1999.

[49] T. Drummond, H. Carim and C. Oster, "Stethoscope with Frictional Noise Reduction". U.S. Patent 7806226, 5 Oct 2010.

[50] S. A. Prasad, Q. Gallas, S. Horowitz and B. Homeijer, "Analytical Electroacoustic Model of a Piezoelectric Composite," in 43rd AIAA Structures, Structural Dynamics, and Materials Conference, Denver, 2006.

[51] S. Hu, R. Rajamani and X. Yu, “Invisible Speakers in Home Windows for Simultaneous Auxiliary Audio Playback and Active Noise Cancellation,” Mechatronics, Vol. 22, No. 8, pp. 1031-1042, December 2012.

[52] X. Yu, R. Rajamani, K.A. Stelson and T. Cui, “Active Control of Sound Transmission Through Windows with Carbon Nanotube Based Transparent Actuators,” IEEE Transactions on Control Systems Technology, Vol. 15, No. 4, pp. 704-714, July 2007.

[53] Y. Wang, R. Madson and R. Rajamani, "Nonlinear Observer Design for a Magnetic Position Estimation Technique," in 2015 IEEE Conference on Decision and Control, Osaka, Japan, December, 2015.

[54] R. Rajamani, “Vehicle Dynamics and Control,” Springer Verlag, second edition, ISBN 9781461414322, January 2012 
[55] Hou, M., A. Clive Pugh, and Peter C. Müller. "Disturbance decoupled functional observers." Automatic Control, IEEE Transactions on 44.2 (1999): 382-386.

[56] Bhattacharyya, S. P. "Observer design for linear systems with unknown inputs." Automatic Control, IEEE Transactions on 23.3 (1978): 483-484.

[57] Tsui, Chia-Chi. "A new design approach to unknown input observers." Automatic Control, IEEE Transactions on 41.3 (1996): 464-468.

[58] G. Ciccarella, M. Dalla Mora and A. Germani, “A Luenberger-Like Observer for Nonlinear Systems,” International Journal of Control, Vol. 57, No. 3, 537-556, 1993,

[59] A. Germani and P. Pepe, European Journal of Control, “A State Observer for a Class of Nonlinear Systems with Multiple Discrete and Distributed Time Delays,” Vol. 11, Issue 3, pp. 196-205, 2005.

[60] Y. Wang, R. Rajamani and D.M. Bevly, “Observer Design for Differentiable Lipschitz Nonlinear Systems with Time-Varying Parameters,” Proceedings of the 2014 IEEE Conference on Decision and Control, December 2014.

[61] G. D. Nelson and R. Rajamani, “Observer Design for Disturbance Input Estimation to Improve Auscultation with a Stethoscope,” in Proceedings of the 2016 American Control Conference, Boston, USA, July 2016.

[62] H.K. Khalil, Nonlinear Systems, $3^{\text {rd }}$ edition, ISBN 0-13-067389-7, Prentice Hall, 2002. 


\section{Side Bar: Observers for Nonlinear Systems with Output Nonlinear Functions}

Although many nonlinear observer design methods have been reported in literature [21], [22], [23], their application is often limited only to systems in which the nonlinearity is contained entirely in the state equation. The output equation is typically assumed to be linear. In the cases where the output equation allows a nonlinear function [20], [58], [59], the constraints on this function do not allow for the inversepower nonlinearities described in the magnetic position sensor application.

The argument cited by researchers in favor of the output linearity assumption is that a coordinate transformation can be used to convert algebraic nonlinear output equations into linear equations. However, a practical investigation shows that using coordinate transformations for the purpose of making output equations linear can be a complicated approach.

Consider the following dynamic model and output equation

$$
\begin{aligned}
& \frac{d}{d t}\left\{\begin{array}{l}
x \\
\dot{x} \\
\ddot{x}
\end{array}\right\}=\left[\begin{array}{lll}
0 & 1 & 0 \\
0 & 0 & 1 \\
0 & 0 & 0
\end{array}\right]\left\{\begin{array}{l}
x \\
\dot{x} \\
\ddot{x}
\end{array}\right\} \\
& y=B(x)=\frac{p\left(2 x^{2}-z^{2}\right)}{2\left(x^{2}+z^{2}\right)^{\frac{5}{2}}}+r
\end{aligned}
$$

This is the type of model that is obtained when the magnetic sensor is placed on top of the cylinder, instead of co-axial with the cylinder, as shown in Figure S1. The kinematic model in equation (S1) is straightforward. The jerk (derivative of acceleration) of the piston position is assumed to be zero. The output (S2) is the measured magnetic field at a sensor location which is a direct algebraic function of the piston positon. Unfortunately, the output equation is nonlinear. Attempting to convert the nonlinear output equation to a linear equation turns out to be non-trivial.

Consider the coordinate transformation (S3) to a new set of state variables:

$$
w=\left\{\begin{array}{lll}
B & \dot{B} & \ddot{B}
\end{array}\right\}^{T} .
$$

With this transformation, the output equation is now linear,

$$
y=B=\left[\begin{array}{lll}
1 & 0 & 0
\end{array}\right] w,
$$


but the state equations are not easy to determine with the new states. Differentiating the variable $B$ and simplifying, the equations in (S5) are obtained,

$$
\dot{B}=\frac{d}{d t}\left(\frac{p\left(2 x^{2}-z^{2}\right)}{2\left(x^{2}+z^{2}\right)^{\frac{5}{2}}}+r\right)=\frac{p(4 x)}{2\left(x^{2}+z^{2}\right)^{\frac{5}{2}}} \dot{x}-\frac{5}{4} \frac{p\left(2 x^{2}-z^{2}\right)}{\left(x^{2}+z^{2}\right)^{\frac{7}{2}}} \dot{x} .
$$

In equation (S5) above, it is very difficult to replace the variables $x$ and $\dot{x}$ with a function of the new states $B, \dot{B}$ and $\ddot{B}$. In order to determine the equation for the dynamics (derivative) of $\ddot{B}$, it is necessary to further differentiate (S5) . However, this does NOT lead to an explicit set of equations for the new state variables. The above conversion process becomes even more challenging when more complicated output equations are involved.

Another difference of the results in this article compared to the nonlinear observer design results presented in [20] is that a simpler way to structure the sector condition on the difference of two differentiable nonlinear functions has been proposed. This avoids having to use another LMI to search for the incremental quadratic constraints.

It should be noted that in the model equation (S2), both the function and its partial derivative with respective to $x$ are bounded, since the variable $z$ is always positive (non-zero). 


\section{Side Bar: Application to Separation of Directional Noise Components in}

\section{Acoustics}

Estimation of directional components of sound: A mixed audio signal can be separated into its directional components using spatially separated, but otherwise identical, dual microphones.

Figure S2 shows a schematic of a building window in which a transparent actuator is used in the window to cancel acoustic disturbances that travel into the room through the window pane. Noise from aircraft is a significant environmental disturbance for buildings close to airports and highways [52]. The use of a transparent acoustic actuator in the window pane can be used to control the noise transmission through the window and make the room quieter [51], [52].

One of the estimation challenges in this active noise control problem is the need to estimate the "reference” signal which is needed for feedforward adaptive noise cancellation. The use of a single microphone to measure the reference signal related to the external disturbance is complicated by the fact that in addition to measuring external aircraft noise the external microphone will also measure noise created by the window actuator itself and noise coming from inside the home, such as music being played in the home. The use of dual microphones can be used to separate the two noise components based on their direction of travel. Using partial differential acoustic model equations and the relationships created by spatially separating the two reference microphones, the estimation of each sound component (decoupling) is possible [51].

\section{Acknowledgements}

The research reported in this article was partly supported by research grants from the US National Science Foundation (NSF Grant CMMI 1562006), the Department of the Army (Cooperative Agreement W81XWH-10-1-1051 ) and 3M’s Infection Prevention Division. The contents of the information herein do not necessarily reflect the position or the policy of the U.S. Government, the U.S. Army, or 3M, and no official endorsement should be inferred. 


\section{Author Biographies}

Rajesh Rajamani obtained his M.S. and Ph.D. degrees from the University of California at Berkeley in 1991 and 1993 respectively and his B.Tech degree from the Indian Institute of Technology at Madras in 1989. Dr. Rajamani is currently Professor of Mechanical Engineering at the University of Minnesota. His active research interests include sensors and control systems for automotive and biomedical applications.

Dr. Rajamani has co-authored over 115 journal papers and is a co-inventor on 12 patent applications. He is the author of the popular book "Vehicle Dynamics and Control” published by Springer Verlag. Dr. Rajamani is a Fellow of ASME, has served as Chair of the IEEE CSS Technical Committee on Automotive Control and on the editorial boards of the IEEE Transactions on Control Systems Technology and the IEEE/ASME Transactions on Mechatronics. He has been a recipient of the CAREER award from the National Science Foundation, the 2001 Outstanding Paper award from the journal IEEE Transactions on Control Systems Technology, the Ralph Teetor Award from SAE, and the 2007 O. Hugo Schuck Award from the American Automatic Control Council.

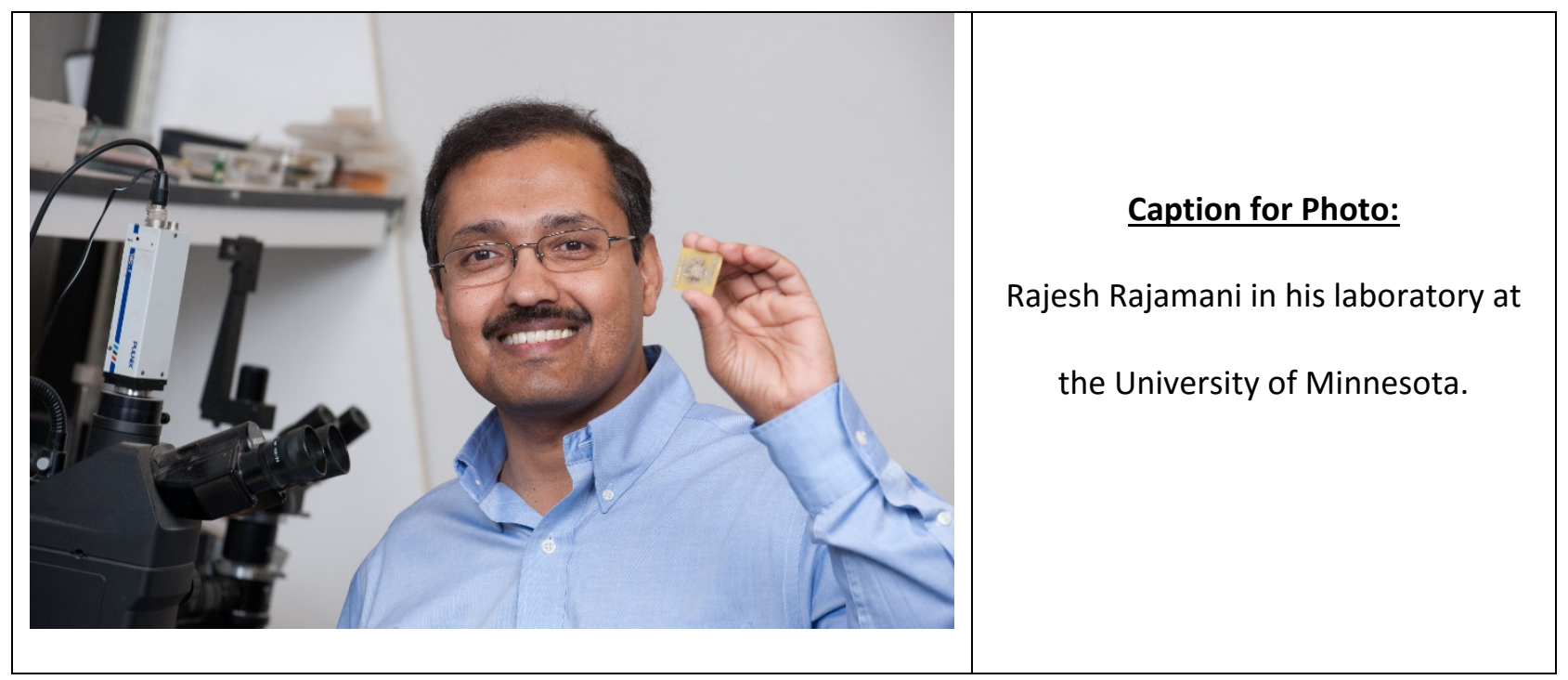




\section{Yan Wang}

Yan Wang earned his M.S from the Delft University of Technology (The Netherlands) in 2006, and his Ph.D. from Auburn University (USA) in 2014, both in Mechanical Engineering. He worked as a controls engineer in TRW Automotive from 2006 to 2009. In Oct. 2014, he joined the Department of Mechanical Engineering at University of Minnesota as a Postdoctoral Research Associate. Yan's research interests include robust control analysis, convex optimization, sensor fusion, and nonlinear observer design.

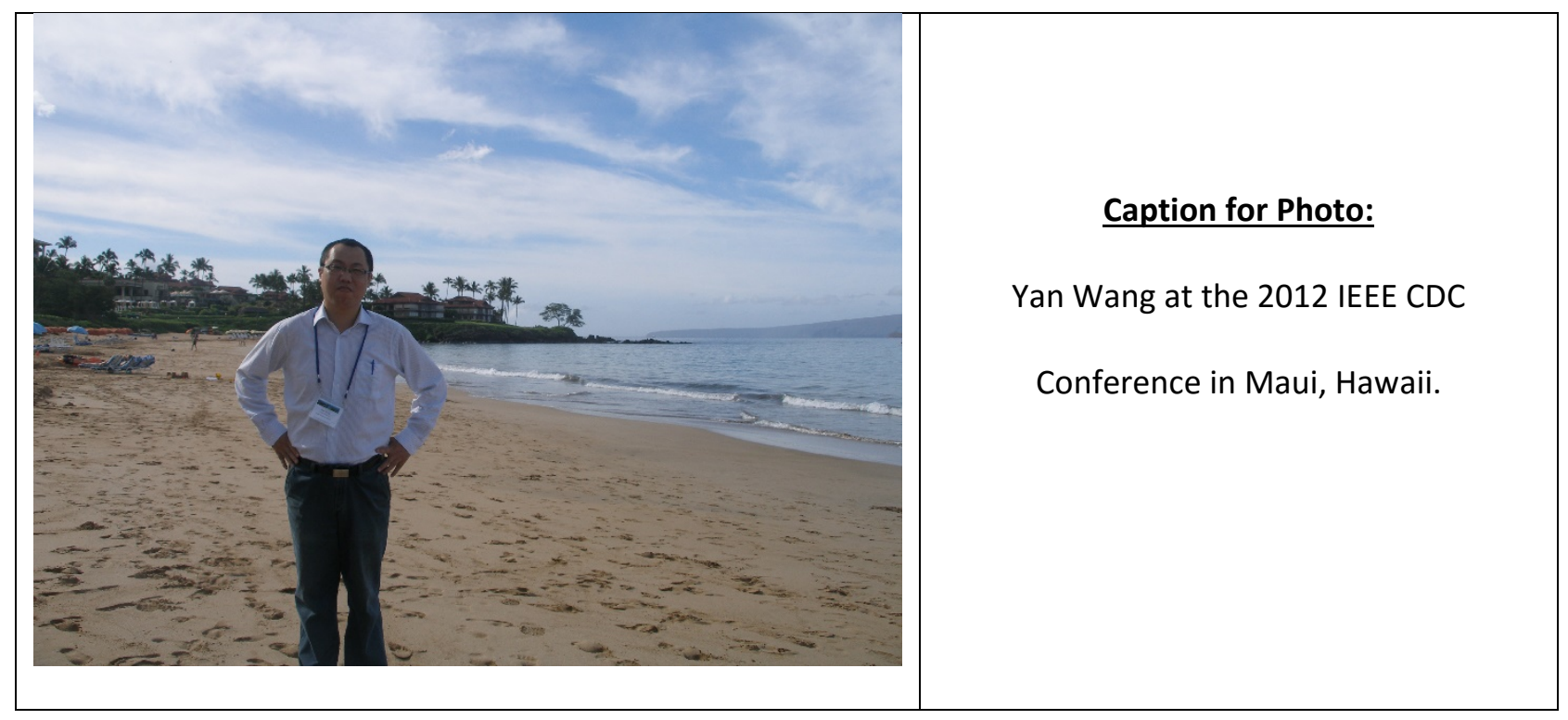

\section{Garrett D. Nelson}

Garrett Nelson received his PhD in Mechanical Engineering and MS in Aerospace Engineering in October 2015 from the University of Minnesota in Minneapolis, MN. He graduated Summa Cum Laude with a BS in Mechanical Engineering in 2010 from the University of St. Thomas in St. Paul, MN. His graduate research was focused on system dynamics with a specialization in estimation and control theory for vibration and acoustic applications. He currently holds a Postdoctoral Appointee position in the Vibration and Acoustic Simulation group at Sandia National Laboratory where he continues research in this area. 


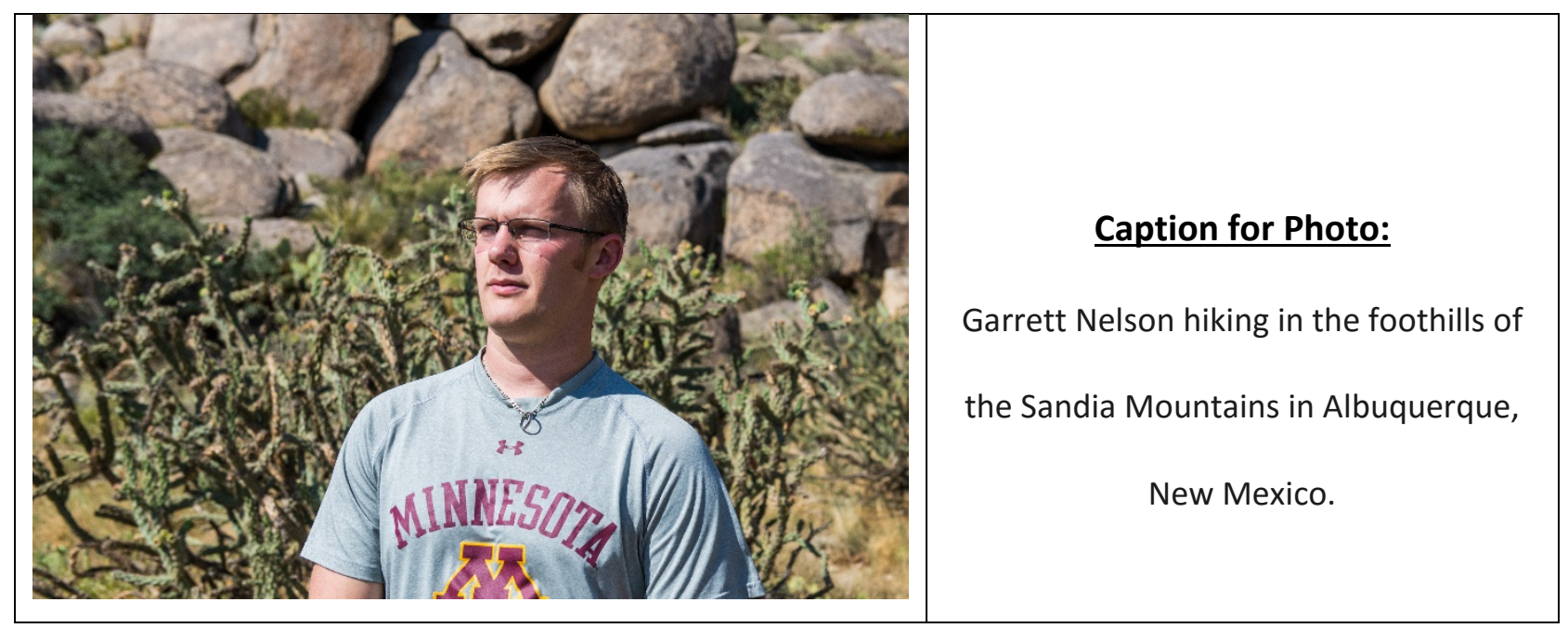

\section{Ryan Madson}

Ryan Madson attended the Massachusetts Institute of Technology for his Bachelor's Degree in Mechanical Engineering. He graduated in spring 2013. Immediately after completing his undergraduate degree, Ryan joined the PhD program at the Department of Mechanical Engineering, University of Minnesota Twin Cities. While completing his studies in the field of controls and estimation, he works at the University as a member of the Laboratory for Innovations in Sensing, Estimation, and Control (LISEC). For his primary research, he is working on developing a novel non-intrusive magnetic field based position sensor. The new technique uses multiple magnetic field sensors to measure the position of an object as well as reject ferromagnetic disturbances. 


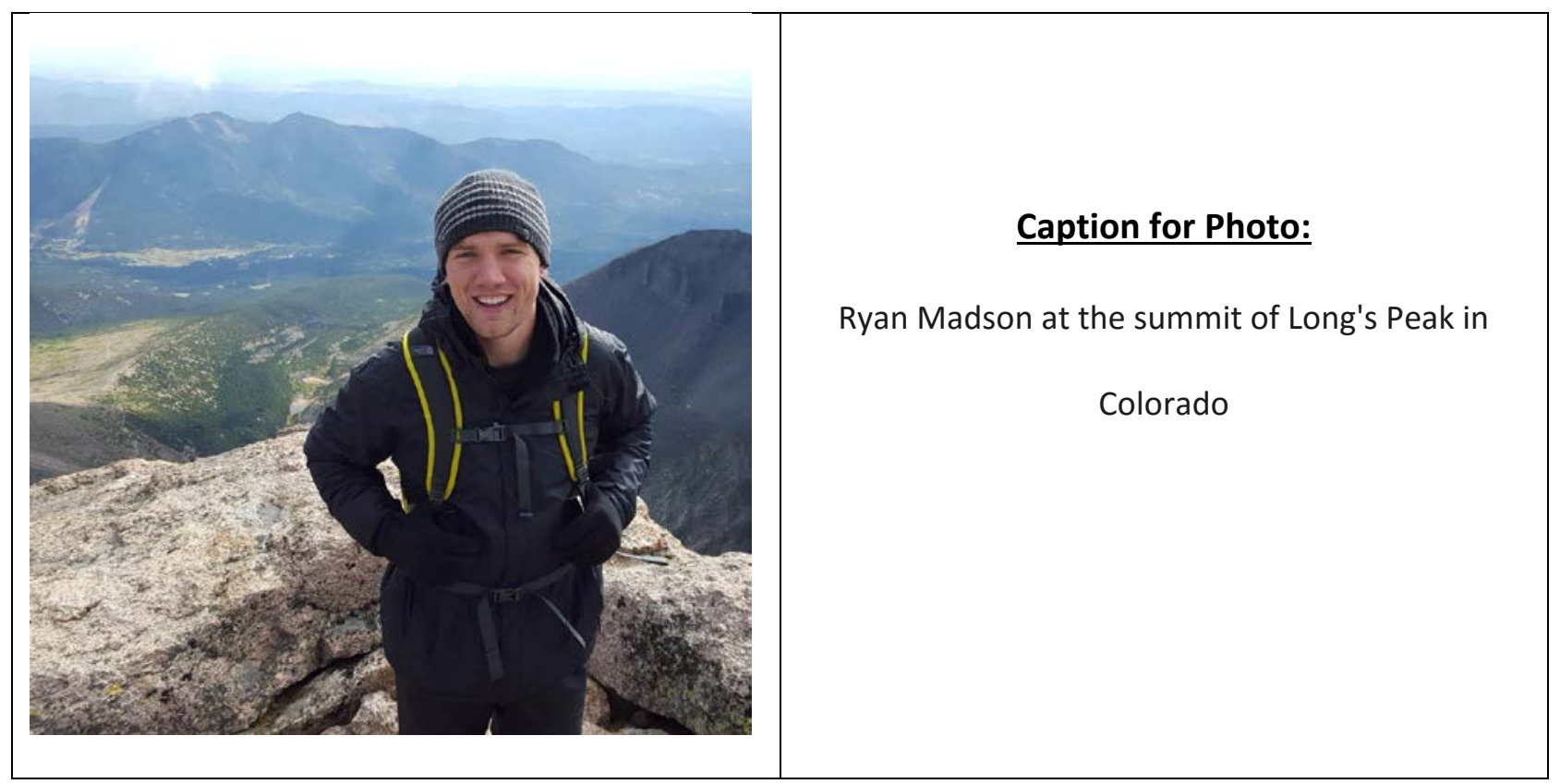

\section{Ali Zemouche}

Ali Zemouche received his Diplôme d’Etudes Supérieures en mathématiques (BS degree in mathematics) from the département des sciences exactes de Oued-Aissi, University Mouloud Mammeri, Tizi-Ouzou, Algeria, in 2000. He also received two Master's degree in mathematics. The first one was received in 2002 from the Institut National Polytechnique de Grenoble, France with specialization in operation research, combinatory and optimization, and the second one was received in 2003 from the University Picardie Jules Verne, Amiens, France, with specialization in applied analysis and modelization. He obtained the Ph.D. degree in automatic control in 2007, from the University Louis Pasteur, Strasbourg, France, where he also held a post-doctorate position from October 2007 to August 2008. Dr. Ali Zemouche has been an associate professor at the Centre de Recherche en Automatique de Nancy (CRAN UMR CNRS 7039) at the University of Lorraine since September 2008. His research activities include nonlinear systems, state observers, observer-based control, time-delay systems, and robustness analysis. Dr. Zemouche is Associate Editor, since 2013, at the IEEE Control Systems Society Conference Editorial 
Board, and is a member of the Technical Program Committee for IEEE-ACC 2017. He is also Associate Editor for European Journal of Control since January 2016.

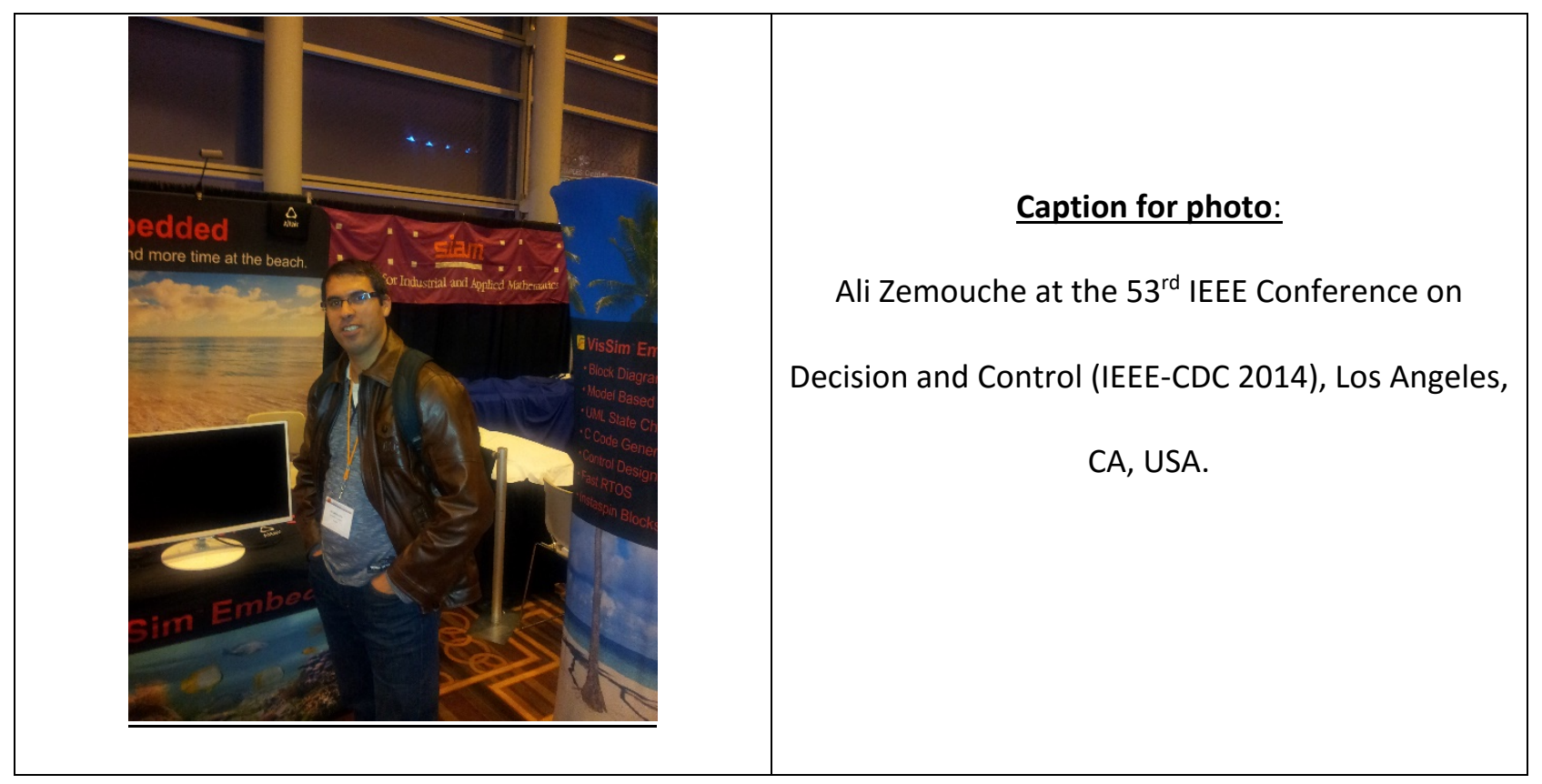




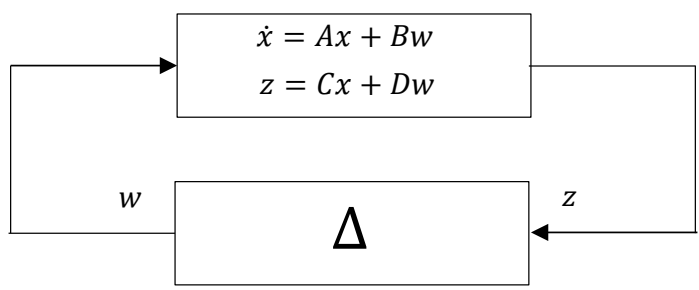

Figure 1. The Lur'e System 


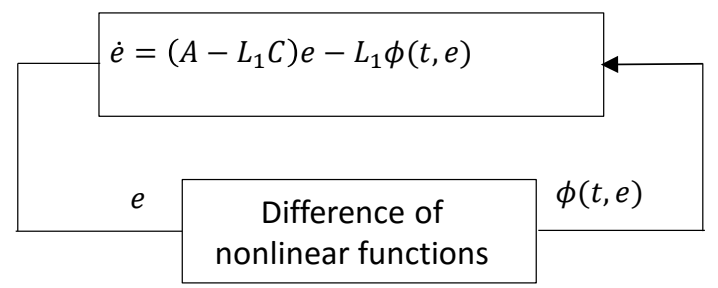

Figure 2. Application of the Lur'e system theory to the observer error dynamics system 


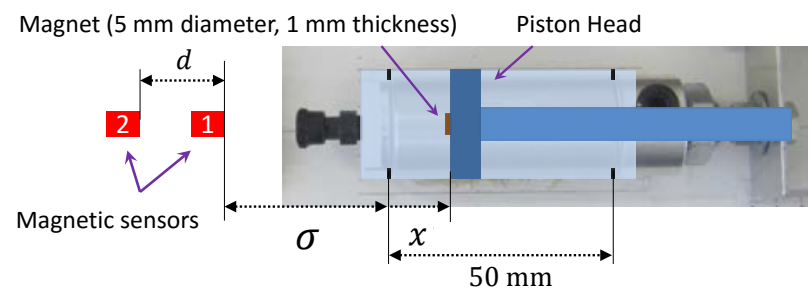

Figure 3. Schematic of a piston in a pneumatic actuator 


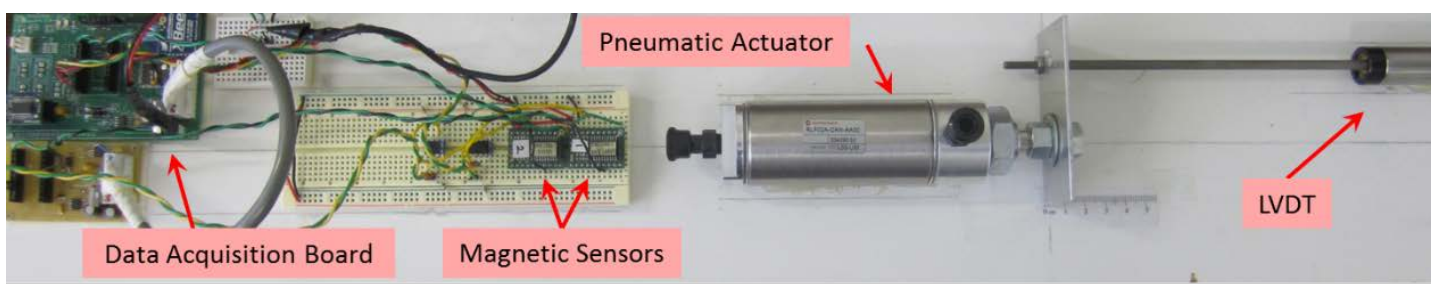

Figure 4. Photograph of a pneumatic actuator and co-axial magnetic sensors 


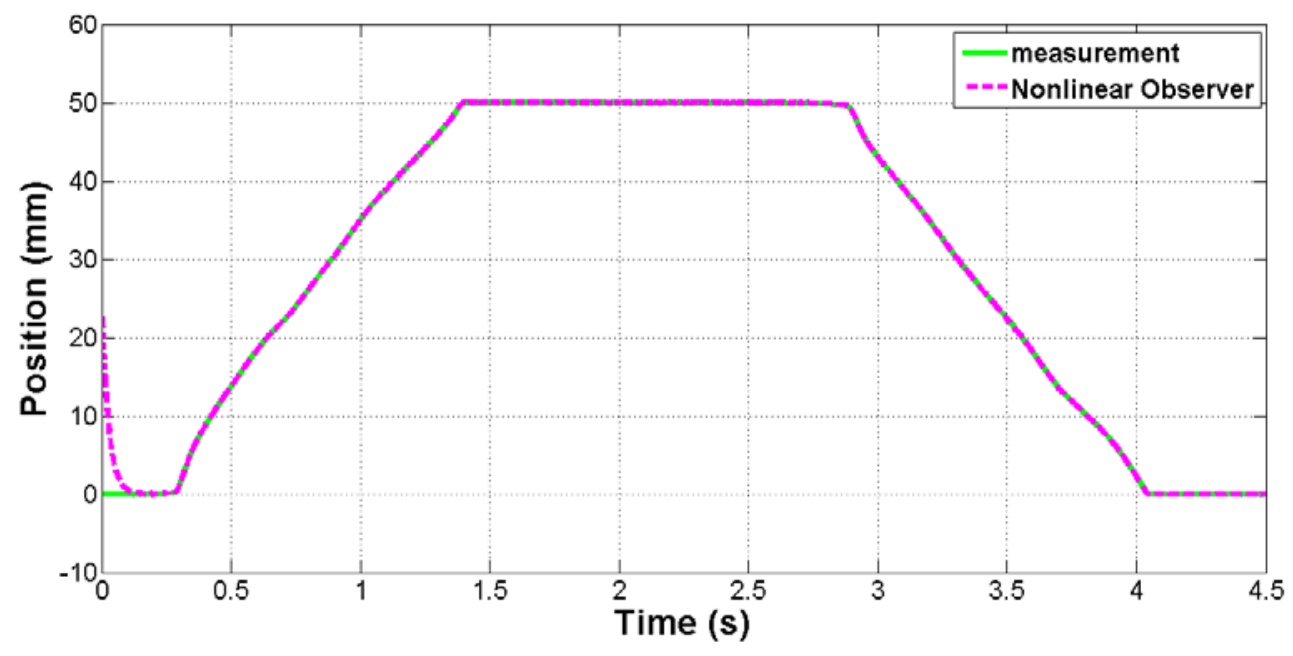

(a) Measured and estimated position profile
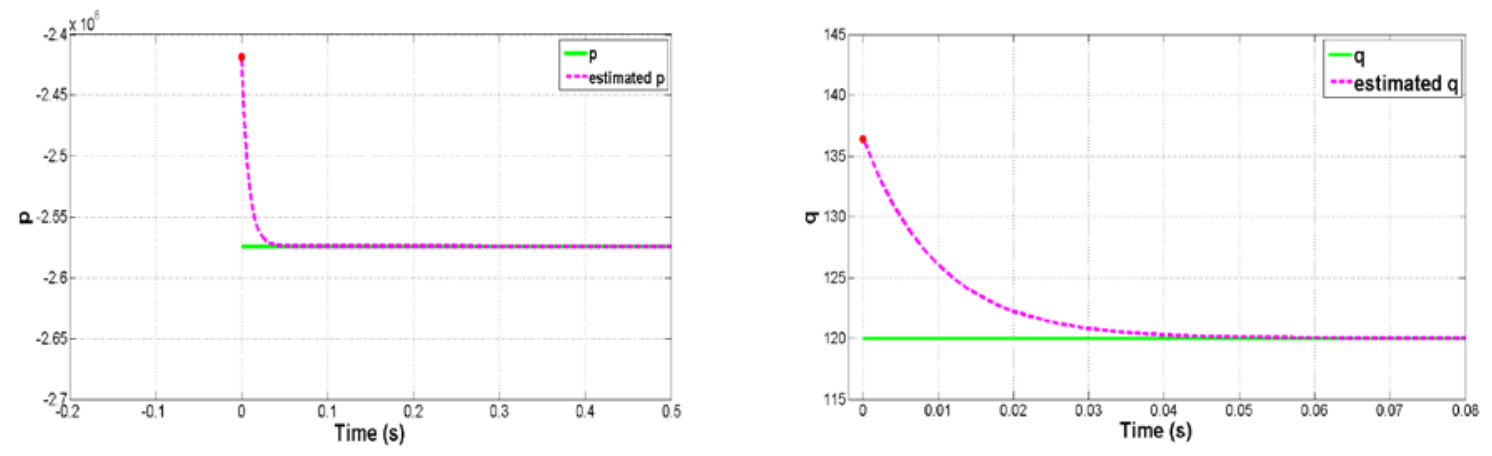

(b) Estimation of parameter $p$ and $\quad$ (c) Estimation of parameter $q$

Figure 5. Experimental results on piston position estimation for a pneumatic actuator using the developed dual sensor observer 

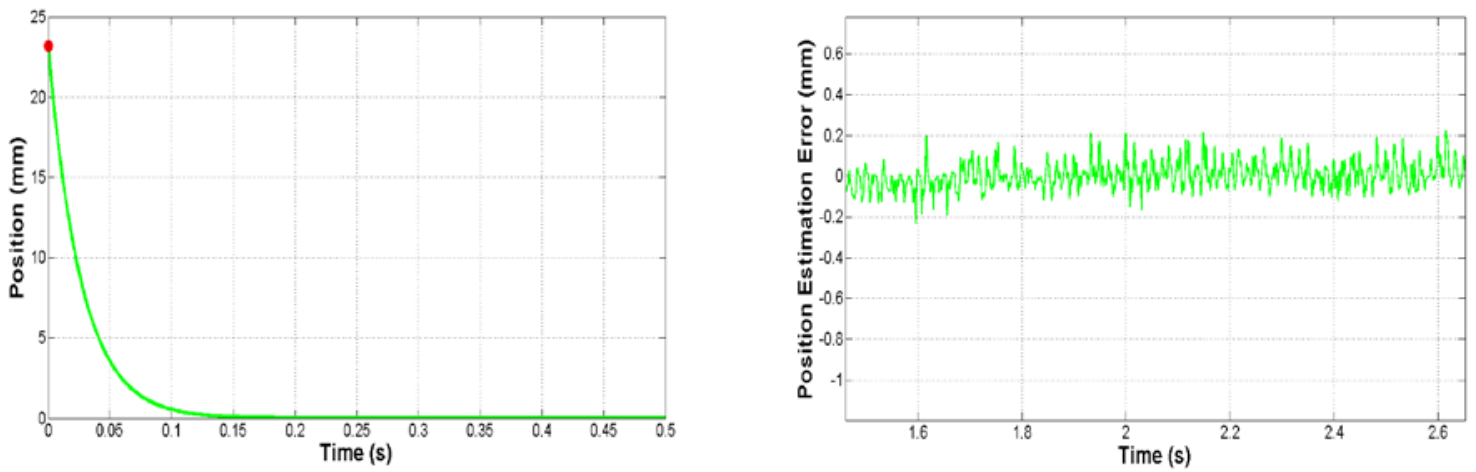

Position estimation error - (a) Transient (b) Steady-state

Figure 6. Performance of observer in experimental results for piston position estimation in pneumatic actuator 


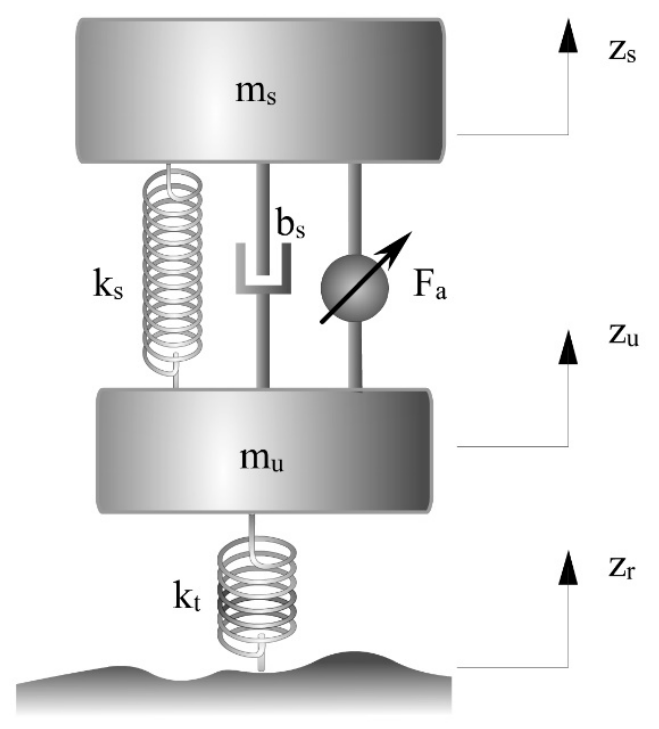

Figure 7. Schematic of a quarter-car automotive suspension 


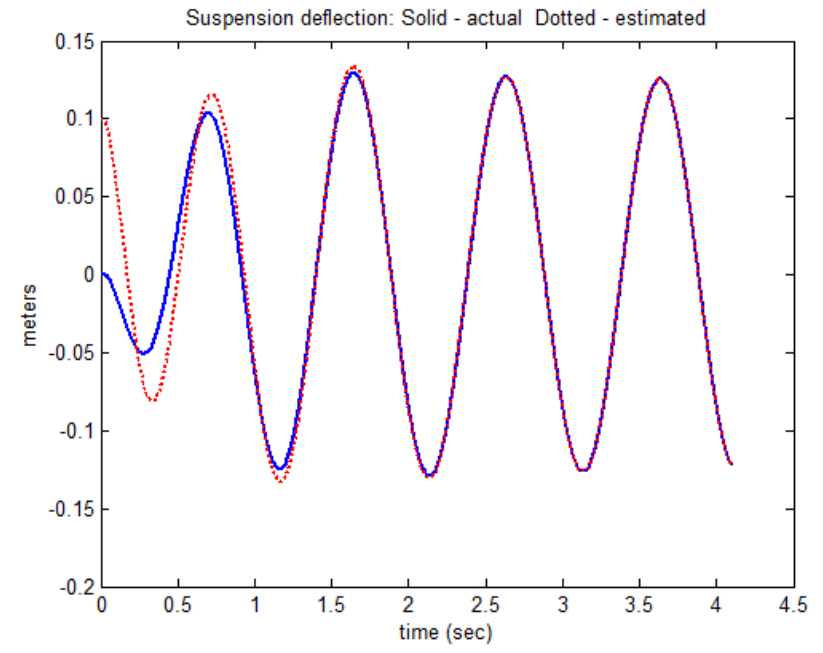

(a) Suspension deflection

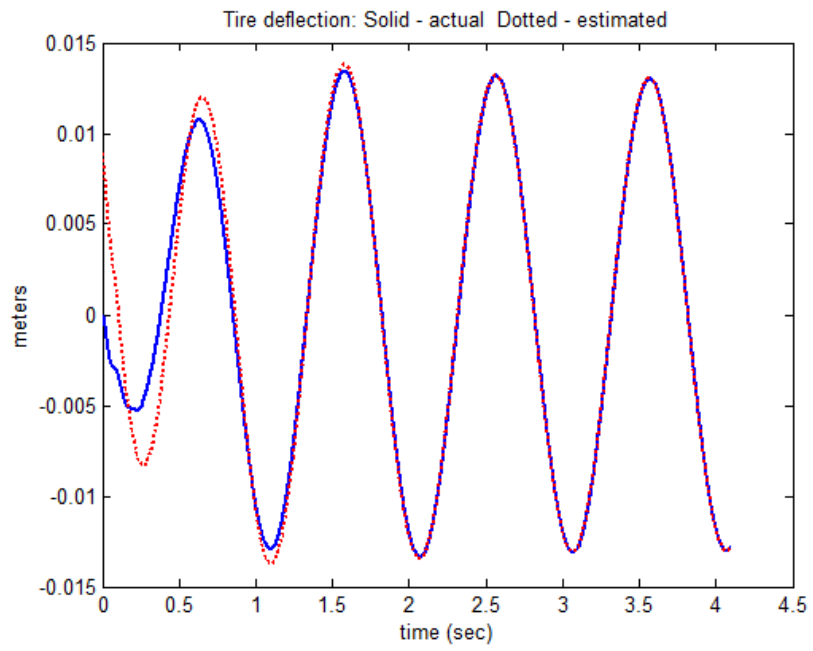

(b) Tire deflection

Figure 8. A comparison of estimates and actual states for a $1 \mathrm{~Hz} \mathrm{road} \mathrm{input}$ 


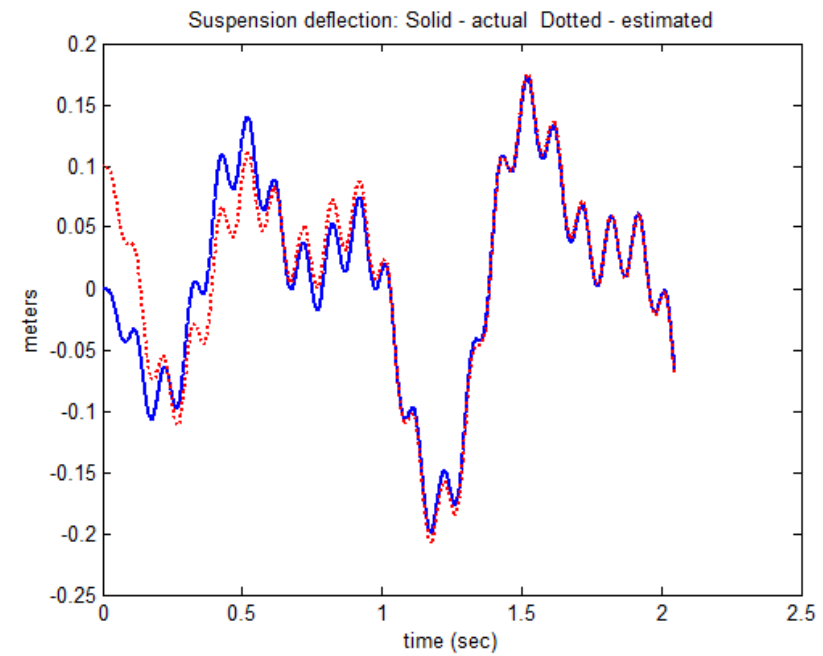

(a) Suspension deflection

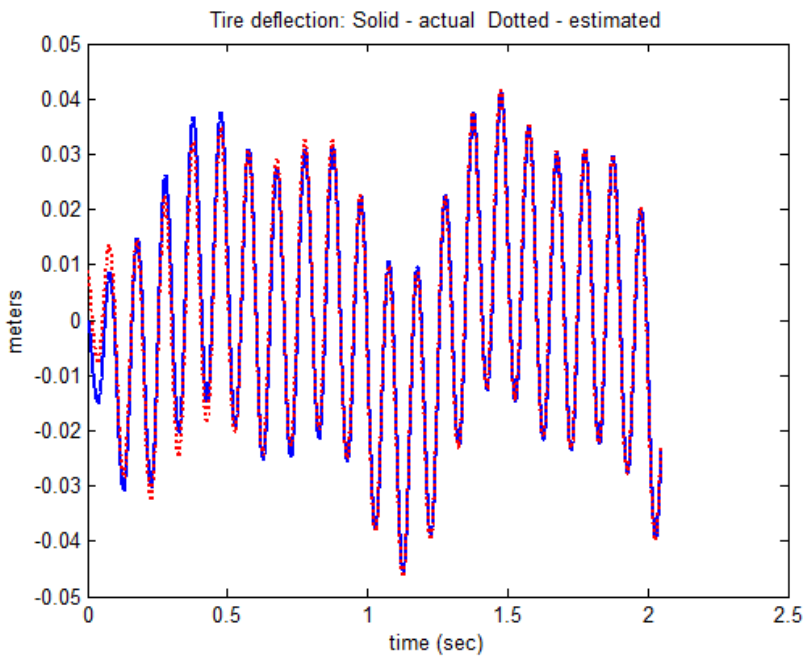

(b) Tire deflection

Figure 9. A comparison of estimates and actual states for a band-limited multi-frequency road input 


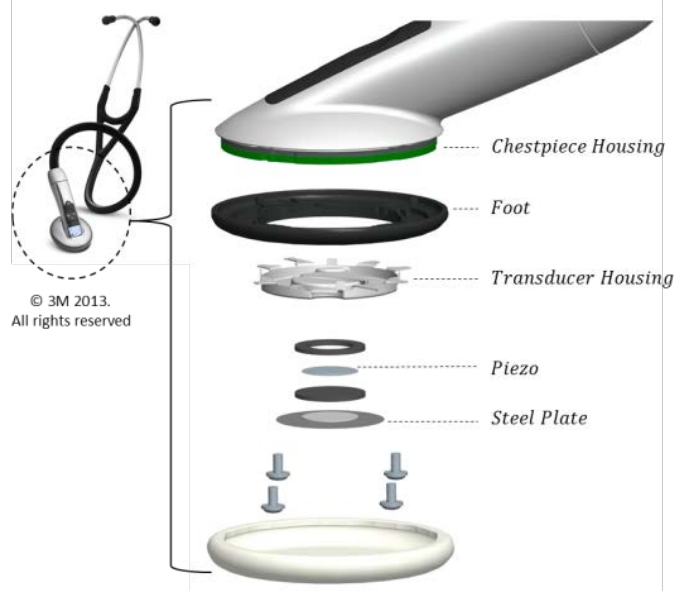

(a) Modern electronic stethoscope and its internal components

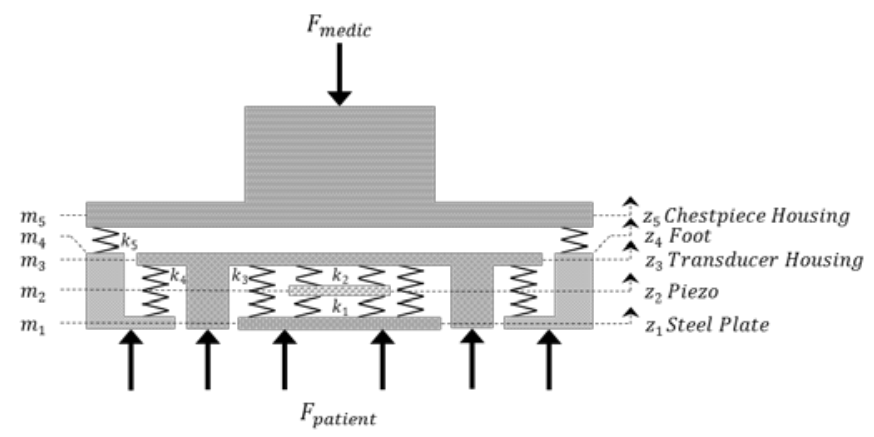

(b) Schematic of the stethoscope for construction of model equations

Figure 10. Schematic illustration of a stethoscope with its internal components and corresponding dynamic model 


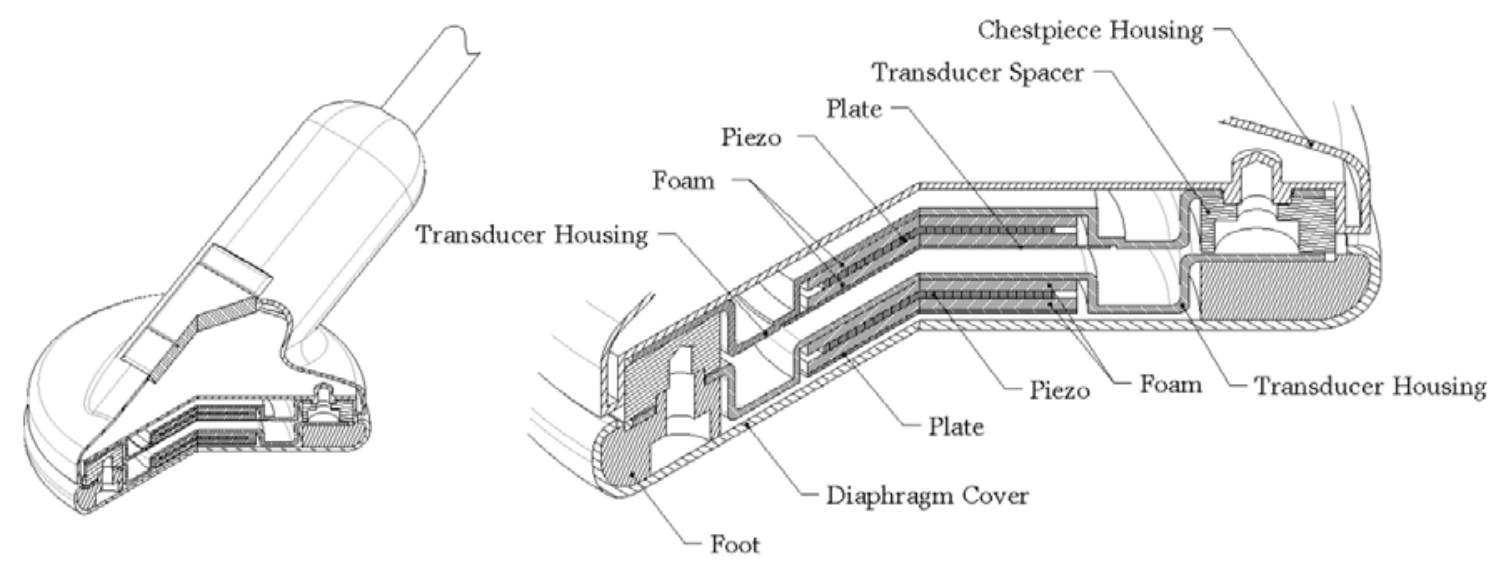

Figure 11. Schematic embodiment of a dual-piezo stethoscope assembly 


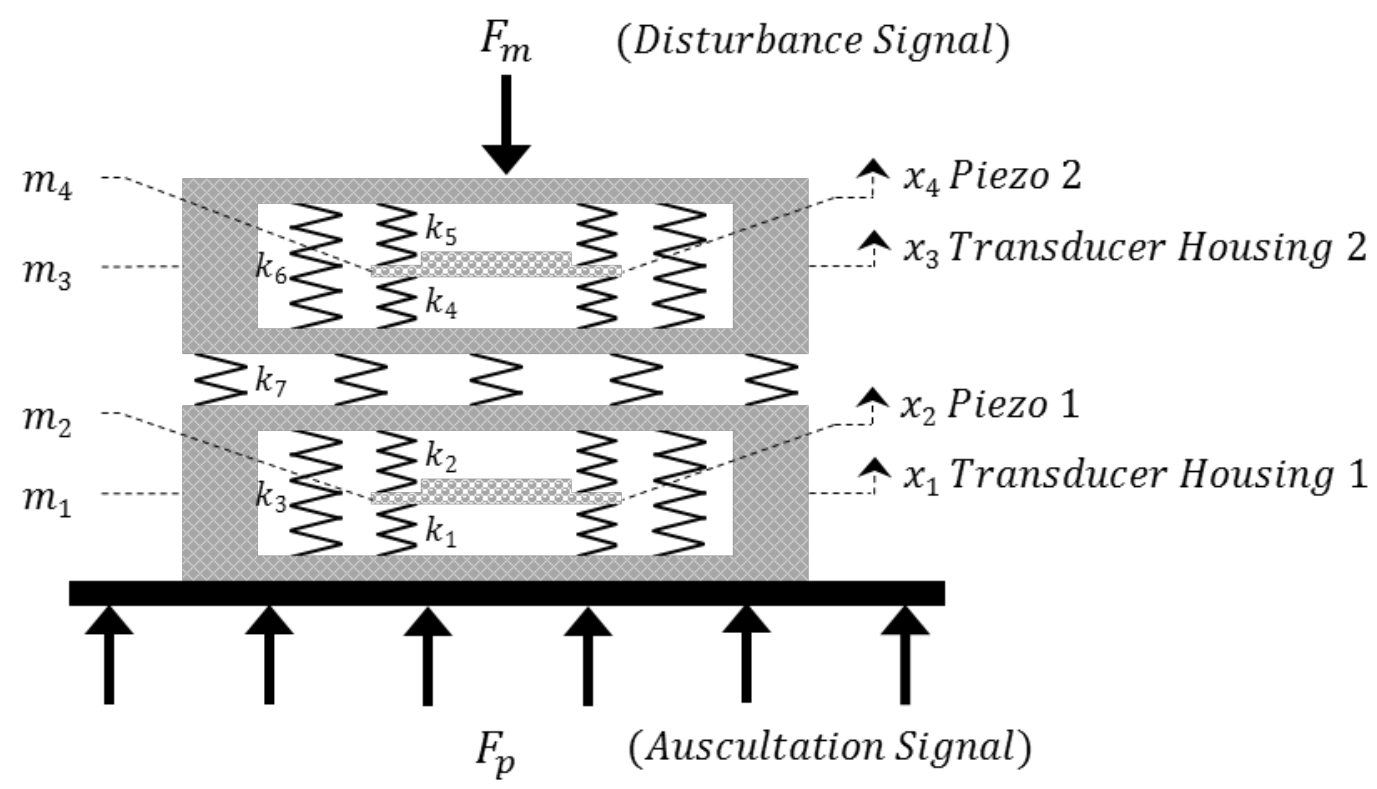

Figure 12. Dual-piezo transducer assembly one-dimensional mechanical model 


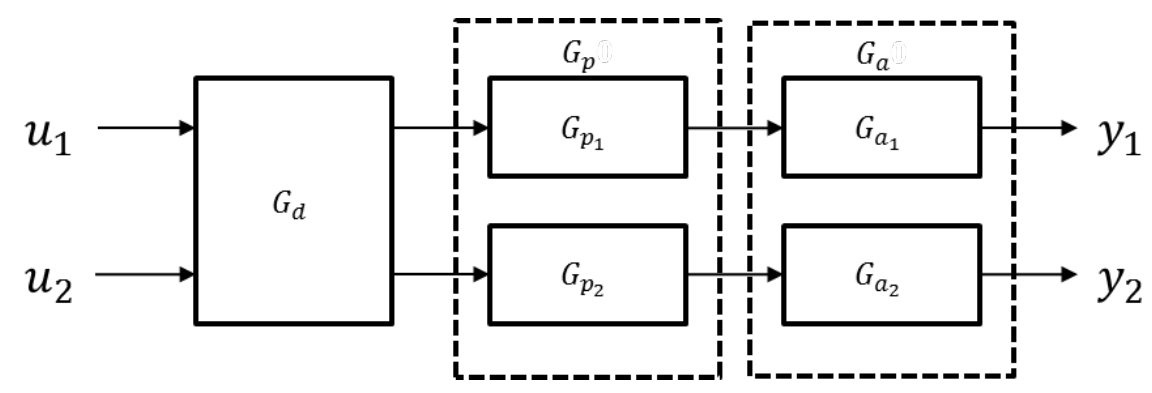

Figure 13. Dual-piezo sensor system model schematic diagram 

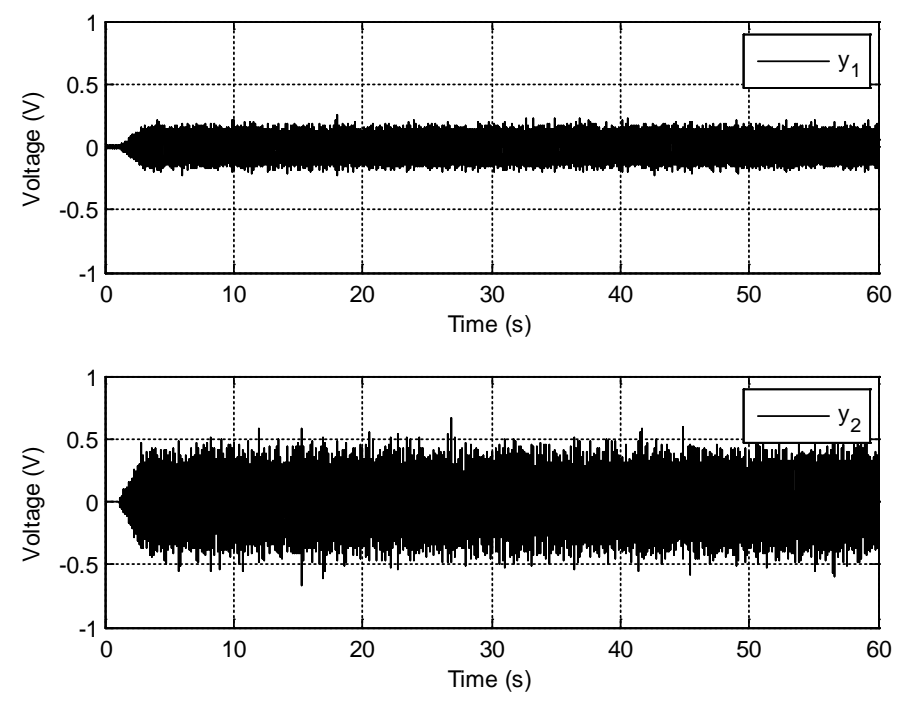

Figure 14. Dual-piezo sensor assembly measurement in presence of unknown vibrational inputs from bottom of assembly only 


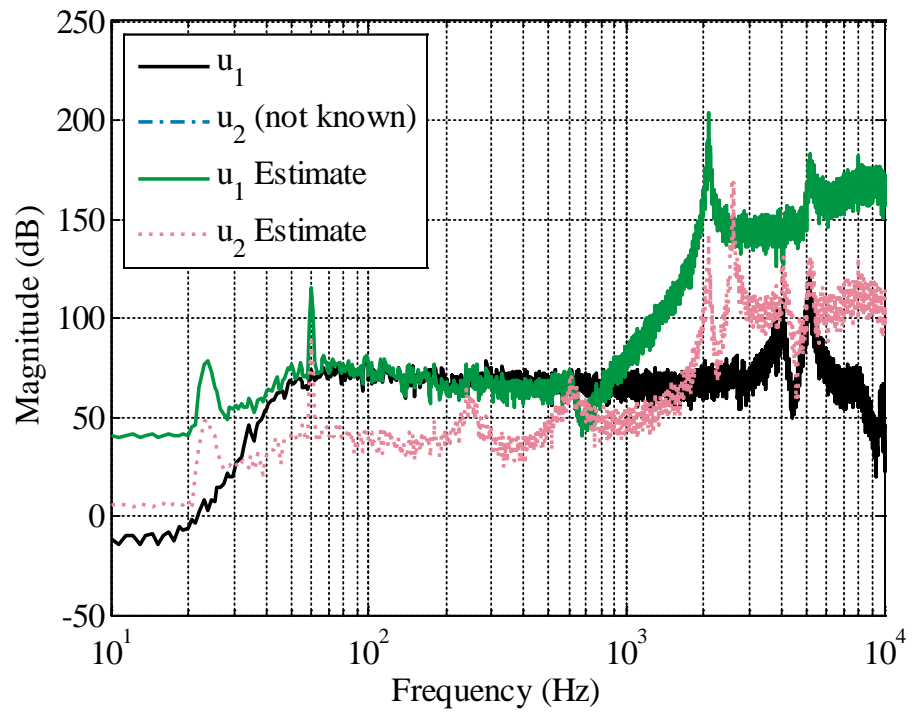

Figure 15. Frequency response of estimate of unknown inputs from a dual-piezo sensor assembly subject to only a white noise patient input 

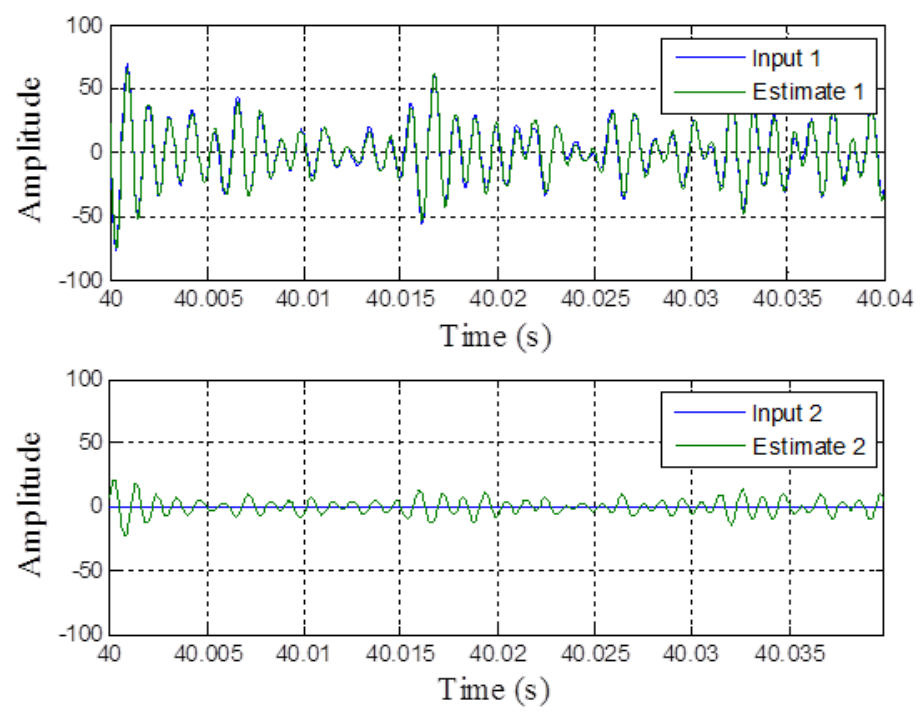

Figure 16. Time series data of estimate of unknown inputs from a dual-piezo sensor assembly subject to only a white noise patient input 


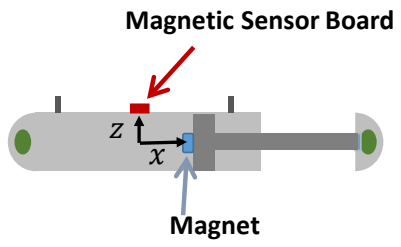

Figure S1. Schematic of a piston-cylinder system with sensor placed on top of cylinder 


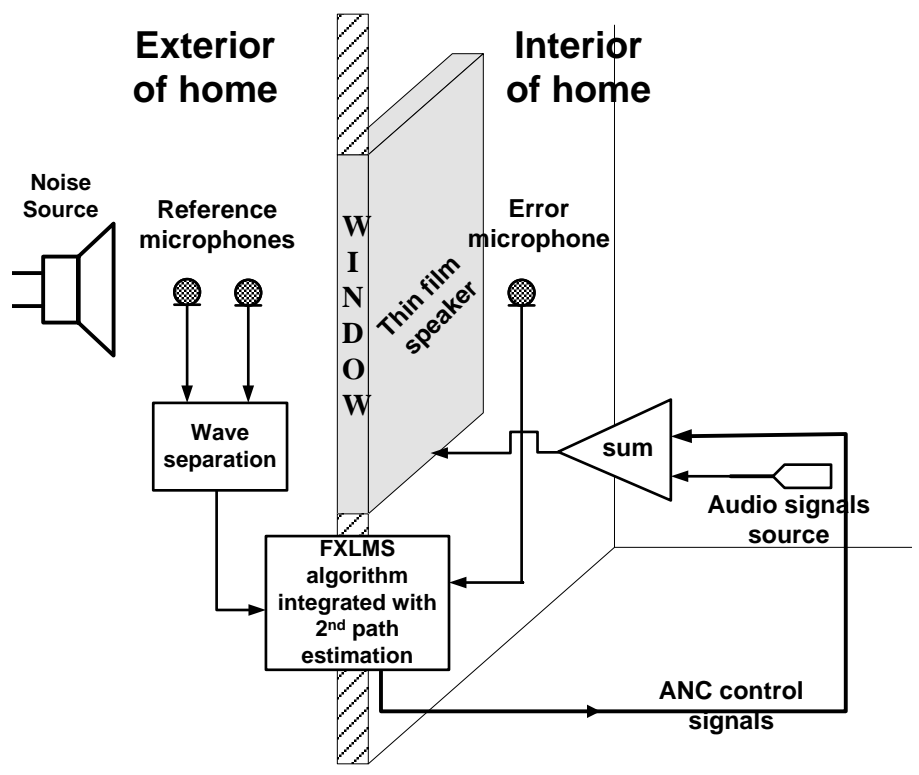

Figure S2. Schematic of a building window, a transparent actuator and microphones 